Shaw, J.O., Briggs, D.E.G., and Hull, P.M., 2020, Fossilization potential of marine assemblages and environments: Geology, v. 49, https://doi.org/10.1130/G47907.1

1 Fossilization potential of marine assemblages and environments

2 Jack O. Shaw ${ }^{1^{*}}$, Derek E. G. Briggs ${ }^{1,2}$, and Pincelli M. Hull ${ }^{1,2}$

3 Department of Earth and Planetary Sciences, Yale University, New Haven, Connecticut

4 06511, USA

$5 \quad{ }^{2}$ Peabody Museum of Natural History, Yale University, New Haven, Connecticut 06520,

6 USA

8 Table of Contents

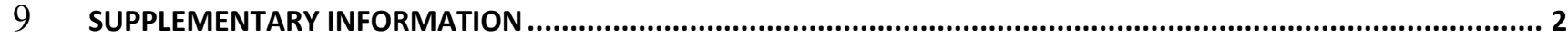

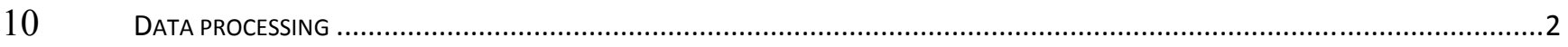

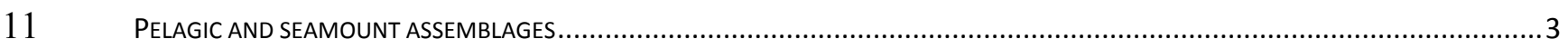

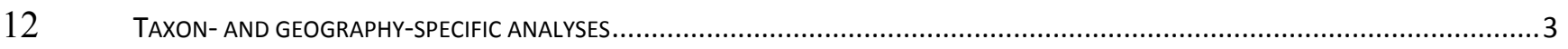

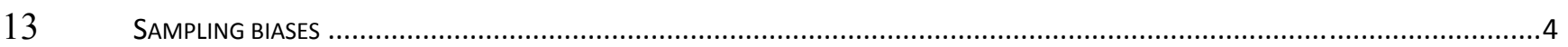

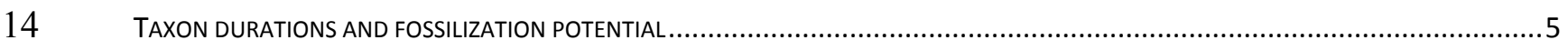

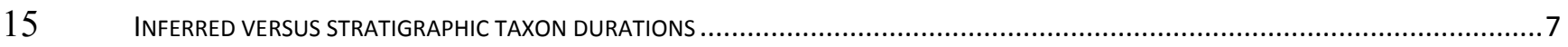

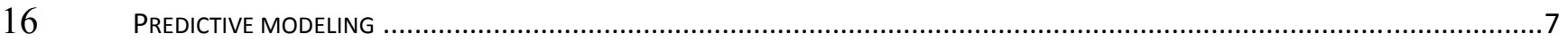

17 IMPACT OF KONSERVAT-LAGERSTÄTTEN ON INFERRED FOSSIIIZATION POTENTIAL OF TAXA.......................................................

18 SUPPLEMENTARY FIGURES ......................................................................................................................10

19 SUPPLEMENTARY DATA DESCRIPTIONS .....................................................................................................29

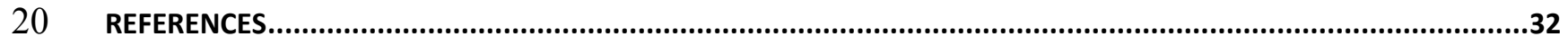

21 


\section{Data processing}

We downloaded all metazoan occurrences from the OBIS on $21^{\text {st }}$ January 2020 (Supplementary Data DR1) and retained only those species names recognized in the World Register of Marine Species (WoRMS) (Horton et al., 2020). We removed occurrences (i) lacking data on genus, latitude, longitude, depth, or duration of sampling, (ii) from non-marine settings, or (iii) with inadequate error control (e.g., citizen science data). Occurrences were grouped into assemblages if they were obtained within $0.1^{\circ}$ latitude and $0.1^{\circ}$ longitude of each other, in the same $10 \mathrm{~m}$ depth bin, during the same year, and from the same dataset. We retained only assemblages with representatives of at least three phyla, four classes, five orders, six families, and seven genera in order to avoid poorly sampled assemblages (Supplementary Data DR2). Alternative binning procedures were tested and did not affect interpretations (Fig. S1). Data processing and analyses were performed in R. All data are provided in the Data Repository (see pages 27-29).

Environmental assignments (shallow $=$ continental shelf and above, deep $=$ continental slope and below, coral reef, and seamount) were based on dataset descriptions and not inferences from faunal lists. We assigned substrate type (mud, sand, gravel, rock) to shallow assemblages ( $\mathrm{n}=$ 7,545 ) based on data interpolated using K-nearest neighbor classification algorithms applied to the dbSEABED database (Jenkins, 2008) (Fig. S6). We did not assign substrates to other environments (e.g., deep water, seamounts, etc.) due to greater uncertainties introduced by the combined effects of lower data densities, interpolation method, and grid size.

44 We downloaded fossil occurrences from the PBDB on $17^{\text {th }}$ January 2020 (Supplementary Data DR3). PBDB occurrences could not be assigned to substrate types due to a lack of sufficient PBDB information on substrate associations. PBDB occurrences were assigned to environments comparable to those in OBIS for the 25,732 genera with accompanying environmental data (Supplementary Data DR6). We used data on Konservat-Lagerstätten (i.e., exceptionally preserved fossil deposits listed in Muscente et al., 2017) which include 20,987 fossil occurrences of 4,398 genera only 208 of which have living representatives recorded in our subset of OBIS occurrences $(n=626,509)$ in order to test their impact on fossilization potential. 


\section{Pelagic and seamount assemblages}

54 In the case of rarely preserved pelagic and seamount assemblages, low within-environment 55 fossilization potentials reflect the rarity of these habitats in the rock record. Neither setting is 56 recorded directly in the PBDB — we had to rely on the presence of 'seamount' and 'pelagic' in

57 the data descriptions associated with PBDB occurrences. We identified seven geologic

58 formations associated with seamounts in the PBDB, containing 483 occurrences and 213 genera.

59 Seventy-eight percent of the OBIS genera exclusive to seamounts $(253$ of 5,635$)$ are represented

60 in the fossil record. Fifty-eight geologic formations in the PBDB are associated with dominantly

61 pelagic taxa, containing 3,437 occurrences and 1,359 genera. Nine percent of the OBIS genera

62 exclusive to pelagic assemblages $(274$ of 5,635) are represented in the fossil record. However,

63 OBIS includes only 6 pelagic datasets (Fig. S8) and they include predominantly fish and/or

64 zooplankton records and omit the abundant gelatinous members of the marine plankton. Thus the

65 true taxon fossilization potential of pelagic environments is likely closer to their within-

66 environment fossil potential of zero than that calculated here (Fig. 3, Fig. S3).

\section{Taxon- and geography-specific analyses}

68 Given the large focus on invertebrates in the PBDB, we performed analyses of fossilization

69 potential excluding vertebrates from OBIS assemblages (Fig. S15). We found minimal changes

$70(<3 \%)$ to mean taxon and within-environment fossilization potential at the global level and when

71 data are parsed by substrate. There are also minimal changes $(<5 \%)$ in shallow, deep, seamount,

72 and pelagic mean fossilization potential values. Coral reefs have higher invertebrate-only taxon

73 (from $44 \%$ to $54 \%$ ) and within-environment (from $26 \%$ to $36 \%$ ) mean fossilization potential

74 values (Fig. S4).

75 In order determine how the spatial distribution of sampling and taxa-for both OBIS and PBDB

76 data - impacted within-environment fossilization potential we analyzed a highly sampled area.

77 Few areas are both well sampled and include assemblages covering a range of environment

78 parameters, but we focused on an area with high PBDB and OBIS coverage, encompassing part

79 of Europe (bounded by coordinates: $58^{\circ} \mathrm{N}, 15^{\circ} \mathrm{W} ; 58^{\circ} \mathrm{N}, 22^{\circ} \mathrm{E} ; 35^{\circ} \mathrm{N}, 15^{\circ} \mathrm{W} ; 35^{\circ} \mathrm{N}, 22^{\circ} \mathrm{E}$ ). Our

80 sampling area contained areas of land and ocean in order to sample fossil and modern diversity.

81 The area contained 8,945 OBIS assemblages, of which 5512 included information allowing

82 within-environment fossilization potential to be calculated. All assemblages with environmental 
83 information in the bounded area were shallow or deep water assemblages. Mean taxon

84 fossilization potential was 34\% and mean within-environment fossilization potential $30 \%$

85 (compared to 38\% and 29\%, respectively, for global data). Mean substrate taxon fossilization

86 potential was lowest in rock and gravel-based assemblages ( $24 \%$ and $20 \%$, respectively) and

87 greatest in sand and mud-based assemblages (33\% and 34\%, respectively), conforming to the same trend of higher fossilization potential in finer grained substrates evident in the global data.

89 Shallow water mean taxon fossilization potential was 33\% and mean within-environment

90 fossilization potential was $31 \%$ (compared to $34 \%$ and $32 \%$, respectively, for global data). Deep

91 water mean taxon fossilization potential was $27 \%$ and mean within-environment fossilization

92 potential was $14 \%$ (compared to $34 \%$ and $15 \%$, respectively, for global data). The greatest

93 difference between the mean fossilization potential of global and European assemblages is the

94 taxon fossilization potential of deep water assemblages, which is $7 \%$ lower in the European

95 sample than the global sample (Fig. S9). This difference is unsurprising, given the large variation

96 in deep water taxon fossilization potential. The consistencies between the European subset and

97 the global data indicate that much of the trend in fossilization potential is robust to geographic

98 sampling biases.

\section{Sampling biases}

100 OBIS assemblages and PBDB taxa are dominated by shallow-water representatives, and these

101 OBIS assemblages generally show the highest fossilization potential values. To test the

102 relationship between sampling and fossilization potential we performed a number of

103 subsampling analyses. It is important to note that the fossilization potential metrics used here are

104 inherently reliant on taxonomic diversity, such that subsampling would remove a key point of

105 our study: the PBDB (and the fossil record) is biased against the fossilization of certain habitats.

106 We tested biases in sampling using the three best sampled groups in terms of number of OBIS

107 assemblages and number of PBDB genera: shallow, coral reef, and deep water environments. We

108 excluded seamount and pelagic communities because subsampling of such small sample sizes-

109 in terms of both OBIS assemblages and PBDB taxa-would obscure any trends. The smallest

110 number of assemblages by environment is $\sim 170$ (seamounts) and the smallest number of fossil

111 taxa by environment is $\sim 200$ (seamounts). 
112 We subsampled to the smallest number of OBIS assemblages by environment (1012, coral reefs)

113 and subsampled to the smallest number of PBDB genera by environment (5927, deep water) to

114 calculate mean fossilization potential values (Fig. S13). We repeated this process 1000 times to

115 generate 1000 mean taxon and within-environment fossilization potential values for each

116 environment. Subsampling data in this way showed that (1) mean taxon fossilization potential

117 values generated using all assemblages (e.g., pictured in Fig. 3) are within the interquartile

118 ranges of mean values of subsampled data (Fig. S13) when parsed by environment, and (2) mean

119 within-environment fossilization potential values generated using all assemblages are greater

120 than mean values of subsampled data due to artificially reduced fossil diversity in the latter, and

121 (3) the larger the reduction in PBDB sample size, the greater the difference between all data

122 mean within-environment fossilization potential values and subsampled data mean values. The

123 within-environment fossilization potential calculation no longer serves its initial purpose of

124 illustrating contrast between PBDB environments, as fossil diversity is maintained for the least

125 sampled environment group but reduced for the other groups.

126 We also varied the number of PBDB taxa sampled by environment (as opposed to sampling to

127 the smallest number of PBDB genera by environment, as described above) in order to consider

128 how biases in fossil sampling impact within-environment fossilization potential values. This

129 showed that, for a given level of fossil sampling, coral reef taxa are proportionally better

130 represented in the PBDB compared to shallow and deep water fossil taxa (Fig. S14).

131 Fossilization potential values at the PBDB sample size of 5927 (Fig. S14, X-axis) are the same as

132 those depicted in within-environment boxplots in Fig. S13.

\section{Taxon durations and fossilization potential}

134 The duration of a taxon impacts the chances of it entering fossil record. A long-lived taxon is

135 more likely to enter the fossil record than a short-ranging taxon as it has a greater chance of

136 recovery. Taxon duration is thought to be determined primarily by geographic range (Jablonski

137 and Hunt, 2006). Additionally proposed biotic and abiotic determinants of duration include

138 habitat depth (Fortey, 1980; Jablonski and Bottjer, 1983), depth range, body size (Jablonski,

139 2008), life mode (Crampton et al., 2010), niche breadth (e.g., number of environments occupied:

140 Kammer et al., 1997). Thus we expect taxon duration to vary between environments and to

141 contribute to assemblage-specific fossilization potential. 
142 We considered the influence of genus duration on fossilization potential in two ways: (1)

143 comparing the durations of OBIS genera recorded in the PBDB between environments and

144 substrates; and (2) including the assemblage-specific means of these durations in a predictive

145 model of genus-level fossilization potential. The mean genus duration of an assemblage only

146 included taxa recorded in the PBDB and excluded taxa without a fossil record.

147 To generate genus durations we utilized the Adaptive Beta method, which is designed to estimate 148 the true stratigraphic range of a taxon based on the temporal distribution of its fossil occurrences 149 (Wang et al., 2016). The code allowed only 150-200 occurrences per taxon to be included. For 150 taxa with more than 150 occurrences in the PBDB we subsampled 150 occurrences (always 151 including the oldest and youngest) 1000 times to generate 1000 estimated origination ages, and 152 used the mean of these ages as the inferred origination age (Data Repository). For taxa with 153 fewer than six occurrences we used the oldest occurrence as the origination age, and for taxa 154 with fewer than 150 occurrences we applied the method without subsampling.

155 We assembled a subset all of the OBIS genera recorded in communities with environmental 156 information $(n=9,669)$. We plotted these data in two forms: (1) boxplots of genus durations by 157 occurrence (Fig. S10A; i.e., genera recorded in multiple OBIS environments were counted 158 multiple times), and (2) boxplots of the assemblage-specific average genus durations (Fig. $159 \mathrm{~S} 10 \mathrm{~B})$.

160 We found that genus durations were similar across substrate types (Fig. S10A). Genus durations 161 were also greatest in shallow water assemblages and lowest in seamount assemblages. When the 162 genus durations of taxa within assemblages were averaged we found that shallow water 163 assemblages have the highest mean values, whereas pelagic and seamount assemblages have the 164 lowest values. Mean assemblage-specific genus durations were greater in finer substrates than in 165 coarser substrates.

166 Using linear regressions, we found a significant - albeit weak — negative correlation between 167 assemblage-specific mean genus duration and taxon fossilization potential ( $\operatorname{slope}=-0.04, \mathrm{p}$ 168 value $<<0.05$, adjusted $\mathrm{R}$-squared $=0.04)$. We also found a significant, weak, positive 169 correlation between mean genus duration and within-environment fossilization potential (slope = 1700.02 , p-value $<<0.05$, adjusted R-squared $=0.01)$. When parsed by environment or substrate we 
171 found variable weakly positive and weakly negative correlations between genus duration and

172 fossilization potential.

\section{Inferred versus stratigraphic taxon durations}

174 The difference between inferred taxon duration (calculated using the Adaptive Beta method) and

175 stratigraphic taxon duration (calculated using the oldest known occurrence in the PBDB) is

176 determined by the relative density of fossil occurrences - the greater the density of occurrences,

177 the smaller the difference between inferred and stratigraphic taxon durations. Thus, any two

178 environments might differ in average inferred taxon duration because either (1) true taxon

179 duration, and thus inferred taxon duration, differs, or (2) true taxon duration is the same but

180 preservation differs, and thus the estimates of inferred taxon duration differ. In the first case we

181 would expect the sites with the best-preserved fossil assemblages to show the largest differences

182 between inferred and stratigraphic durations. In the second case we would expect the best-

183 preserved sites to show the smallest differences between inferred and stratigraphic durations.

184 We find that the difference between inferred genus duration and stratigraphic duration are

185 greatest in shallow environments, followed by coral reefs and deep water environments,

186 seamounts, and pelagic environments (Fig. S16). In other words, differences are greatest in the

187 best-preserved environments, suggesting that we are identifying true differences in taxon

188 durations between environments, rather than differences generated by sampling artifacts.

189 Therefore we used inferred taxon duration as a proxy for true taxon duration in our analyses.

\section{Predictive modeling}

191 We used conditional inference classification trees (CTree) built in the R package partykit

192 (Hothorn and Zeileis, 2015) to assess the relative importance of environmental differences in

193 preservation potential (variables: environment, water depth, substrate, realm, alpha diversity),

194 taxon longevity (variables: average genus duration), and sampling biases (variables: latitude,

195 longitude, alpha diversity) on the fossilization potential of marine assemblages (Data

196 Repository). CTree is a type of Classification and Regression Tree (CART) analysis that

197 iteratively tests subsets of data until the null hypothesis of interdependence among variables

198 cannot be rejected. Unlike some other classification tree models, it accounts for covariation

199 among predictor variables for unbiased selection and identification of variable importance. 
200 Additionally, the model was chosen because it handles overfitting, non-linear and non-

201 parametric data, and missing information. Models were built using only communities with

202 environmental data (i.e., shallow, coral reef, deep water, seamount, and pelagic communities; $n=$

203 9,738). We ran CTree analysis 1000 times for both types of fossilization potential. Relative

204 variable importance scores, R-squared (R2), and root mean square error (RMSE) values were

205 then averaged across the 1000 runs. Each run utilized a random sample of $70 \%$ of all

206 assemblages. Variable significance was assessed at an alpha of 0.05 using Bonferroni adjusted

207 Monte-Carlo p-values (10 permutations per analysis).

208 The dominance of spatial variables (longitude and latitude) in predictive models of fossilization

209 potential is indicative of sampling bias. This is most obvious in analyses of pelagic community

210 fossilization potential where different datasets focus on different taxa with very different

211 fossilization potentials (Fig. S8). However, this sampling bias does not necessarily erroneously

212 skew fossilization potential calculations. Most datasets are inherently spatially and

213 environmentally restricted (i.e., one research group will focus on a small spatial patch, and not

214 sample the globe), and these attributes covary (e.g., the presence of coral reefs around Australia).

\section{Impact of Konservat-Lagerstätten on inferred fossilization potential of taxa}

216 Taxa in Konservat-Lagerstätten add significant data to diversity and disparity estimates and our

217 understanding of macroevolution particularly in the early Paleozoic (Briggs et al., 1994; Hou et

218 al., 2017). Thus Konservat-Lagerstätten might be expected to have a dramatic effect on

219 inferences of fossilization potential by providing rare glimpses of ancient assemblages. However,

220 just $2.2 \%$ of the 58,870 genera in the PBDB are known only from Konservat-Lagerstätten.

221 Furthermore, exceptionally preserved assemblages are much rarer in the Cenozoic (7 Konservat-

222 Lagerstätten), when $75 \%(n=1,921)$ of OBIS genera with PBDB occurrences are found first, than

223 in the Paleozoic (142 Konservat-Lagerstätten). Only 6 genera $(0.05 \%, n=9,806)$ ( 7 families and 3

224 orders) in OBIS are recorded only in Konservat-Lagerstätten deposits and not in other PBDB

225 deposits. When they are omitted from calculations of fossilization potential (i.e., limiting the

226 study to 'typical' preservation conditions), there is no difference in our findings (Fig. S1). Thus

227 our calculations of fossilization potential are robust to the inclusion (or exclusion) of Konservat-

228 Lagerstätten from the PBDB - the shelly fossil record provides the primary archive of potential 
229 assemblage composition. Particularly in the Cenozoic, there are few Konservat-Lagerstätten to 230 fill known gaps in these biased archives. 
232 SUPPLEMENTARY FIGURES

233 

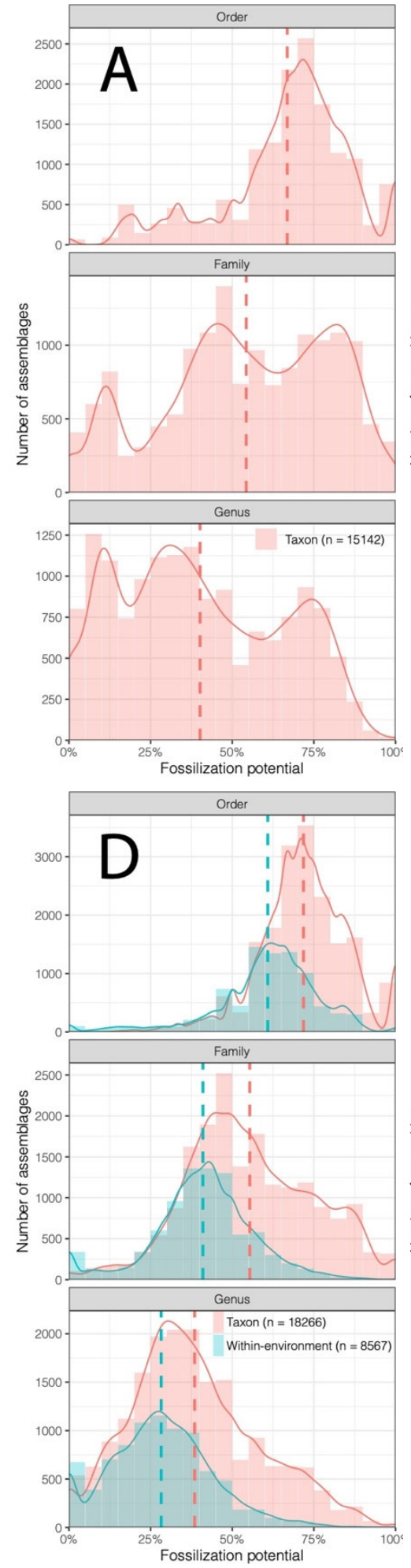
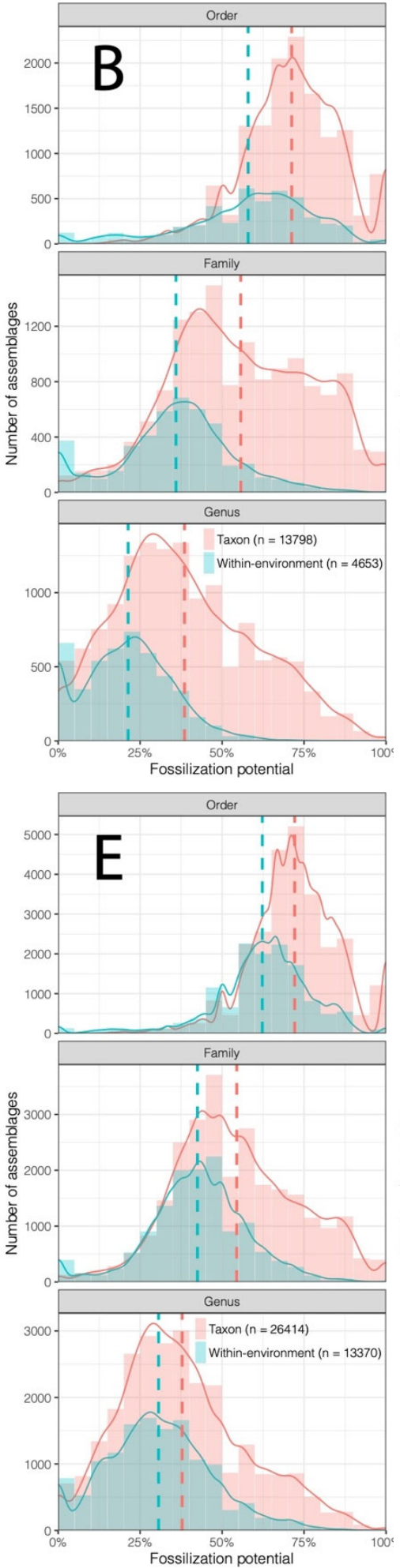
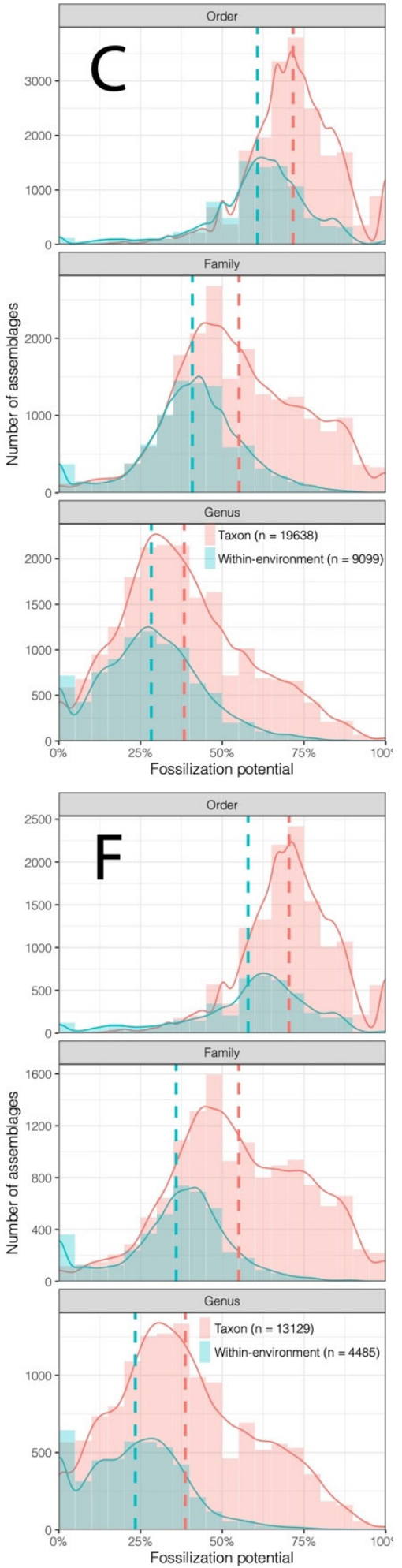

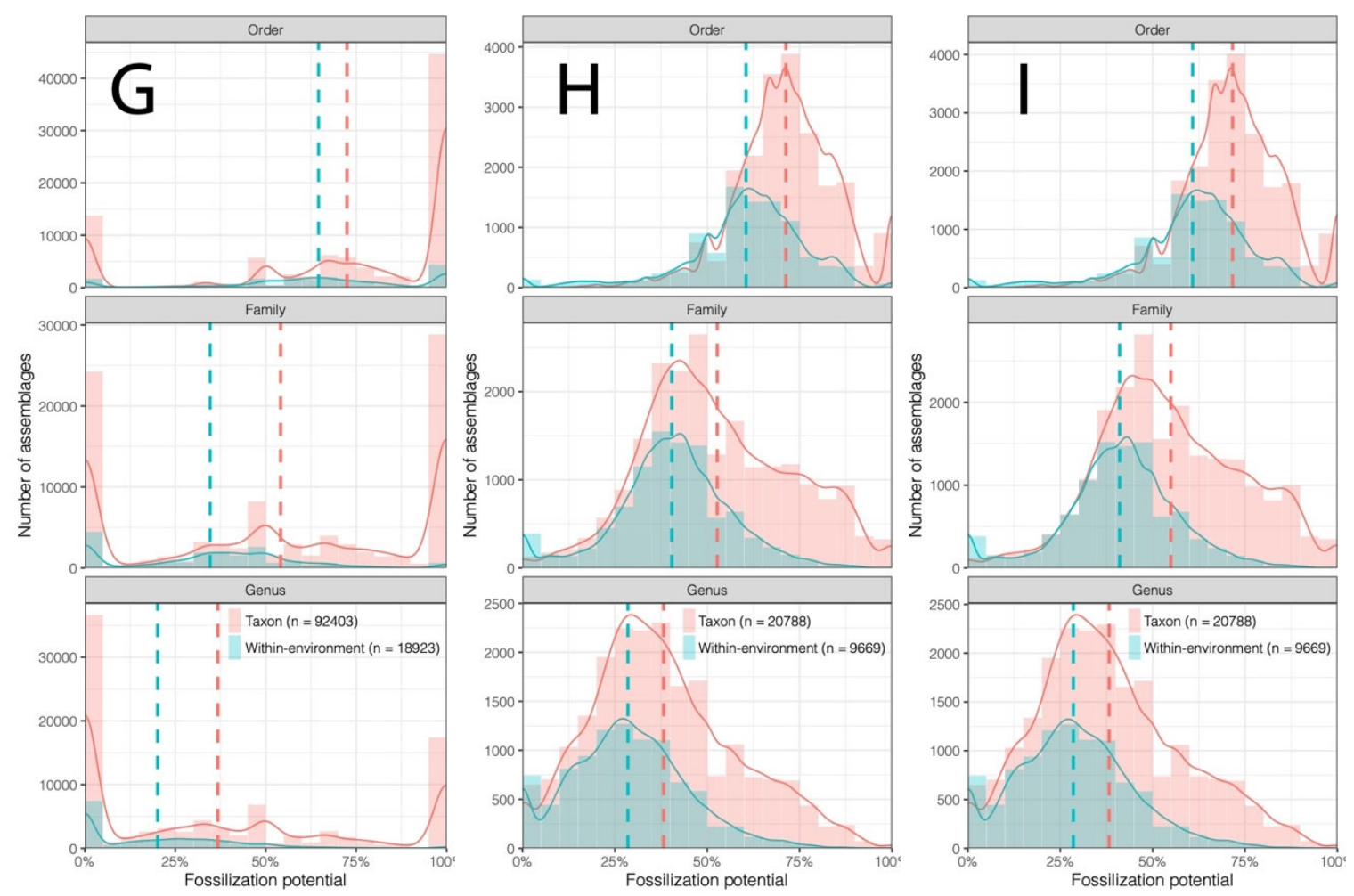

237 Supplementary Figure S1: Comparisons of taxon and within-environment fossilization

238 potential distributions calculated using alternative binning procedures. Unless otherwise noted

239 distributions include the default criteria used in the main text: all PBDB data and OBIS

240 assemblages grouped by $10 \mathrm{~m}$ depth bins, $0.1^{\circ}$ latitude-longitude bins, sampling year, and a

241 minimum diversity of three phyla, four classes, five orders, six families, and seven genera.

242 Assemblages grouped with (A) $20 \mathrm{~m}$ depth bins and $1^{\circ}$ latitude-longitude bins, but not by dataset

243 ID); (B) year excluded; (C) $20 \mathrm{~m}$ depth bins; (D) $100 \mathrm{~m}$ depth bins; (E) $0.01^{\circ}$ latitude-longitude

244 bins; (F) $1^{\circ}$ latitude-longitude bins; (G) no minimum diversity criteria; (H) Lagerstätten

245 excluded; (I) assemblages grouped using the default criteria (Genus panel: Fig. 1, main text). 

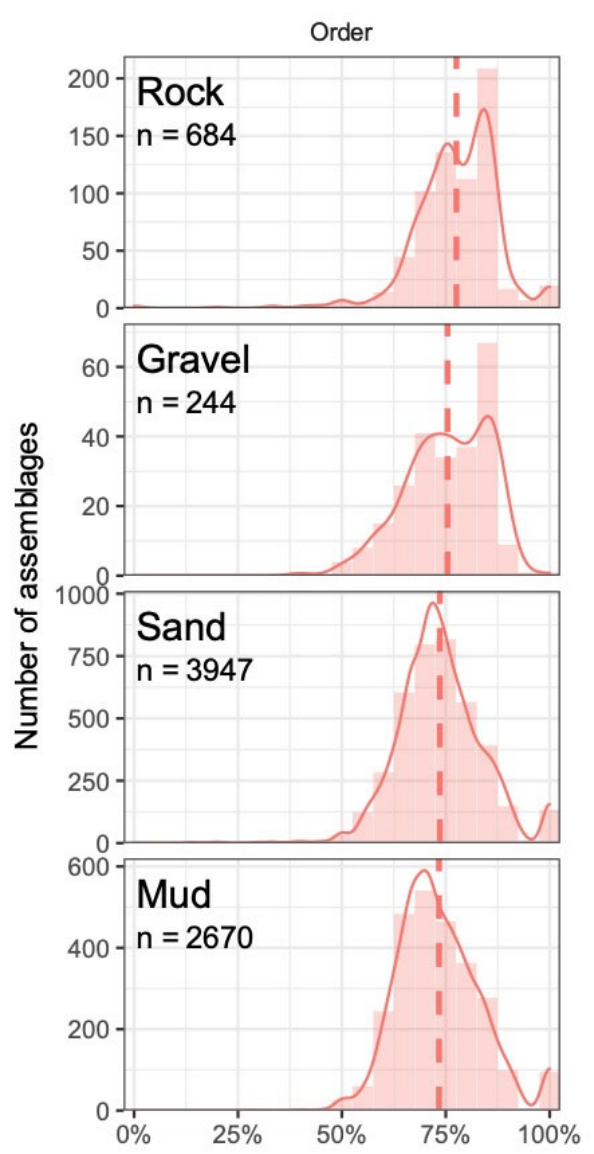

248

249 Supplementary Figure S2: Taxon fossilization potential distributions for shallow-water

250 assemblages with substrate information $(n=7,545)$. Taxon fossilization potential calculated at

251 ordinal, family, and generic ranks. Histogram bin widths $=5 \%$, dashed lines indicate mean.
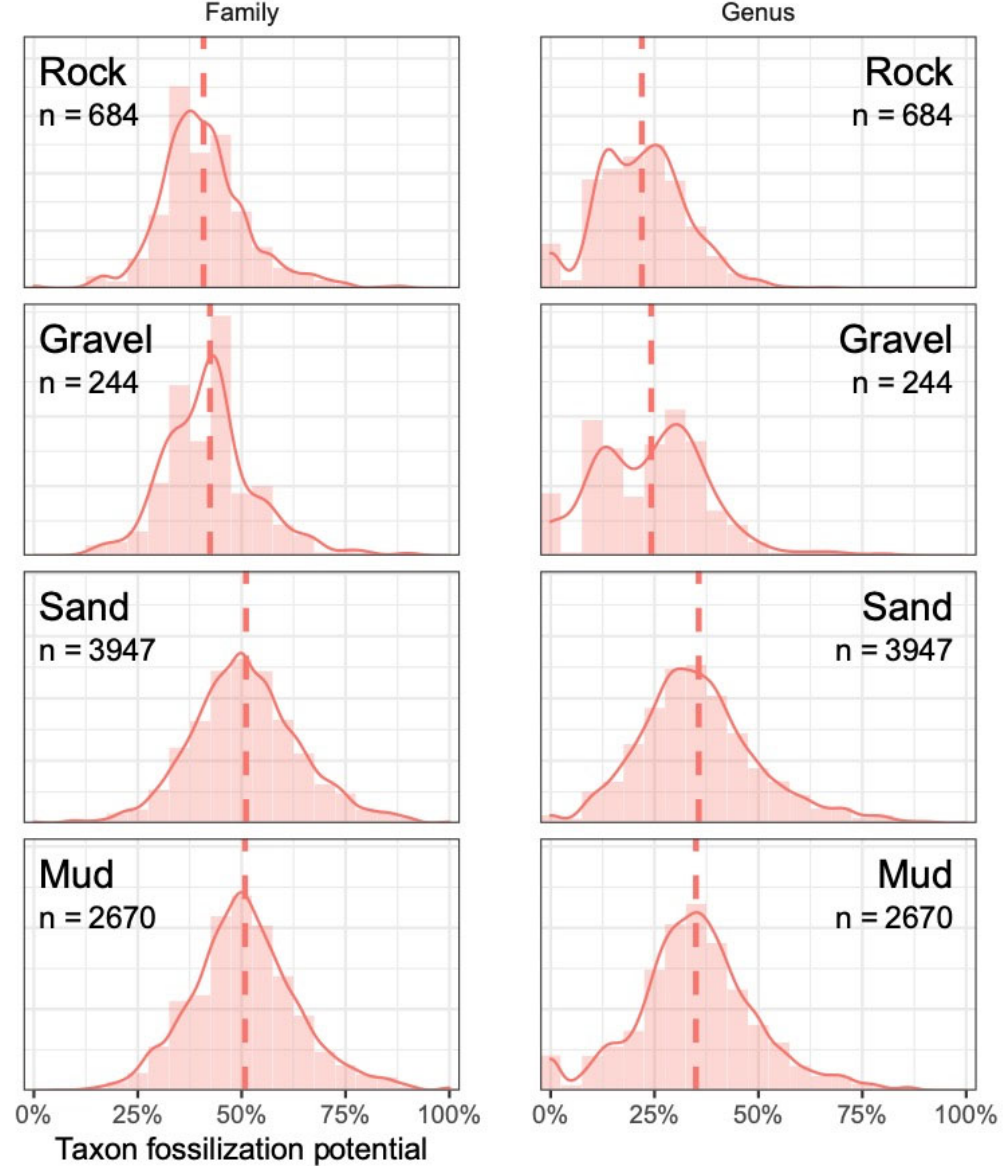

Sand
$=3947$

Taxon fossilization potential 


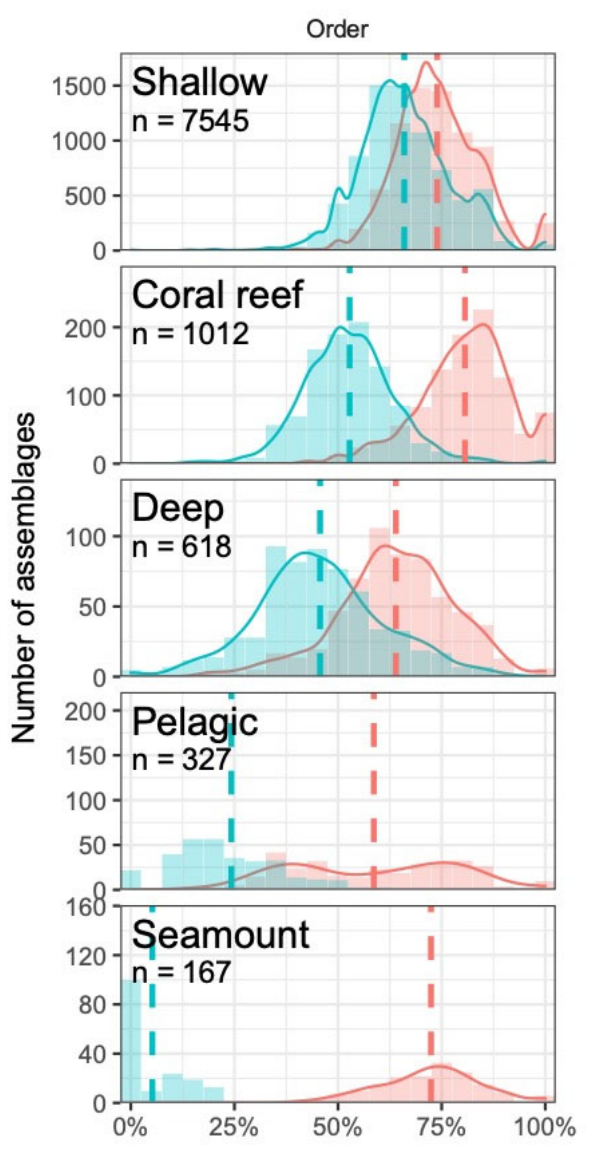

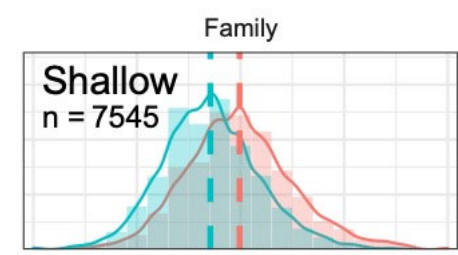
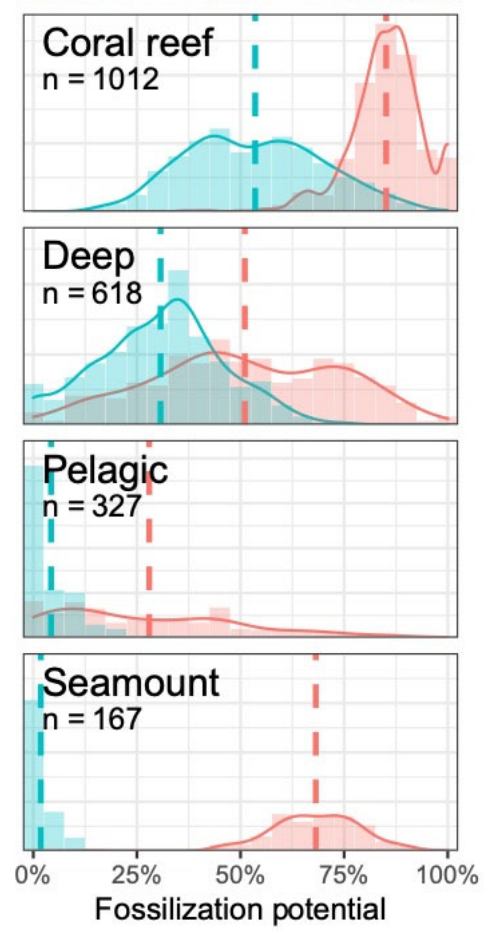
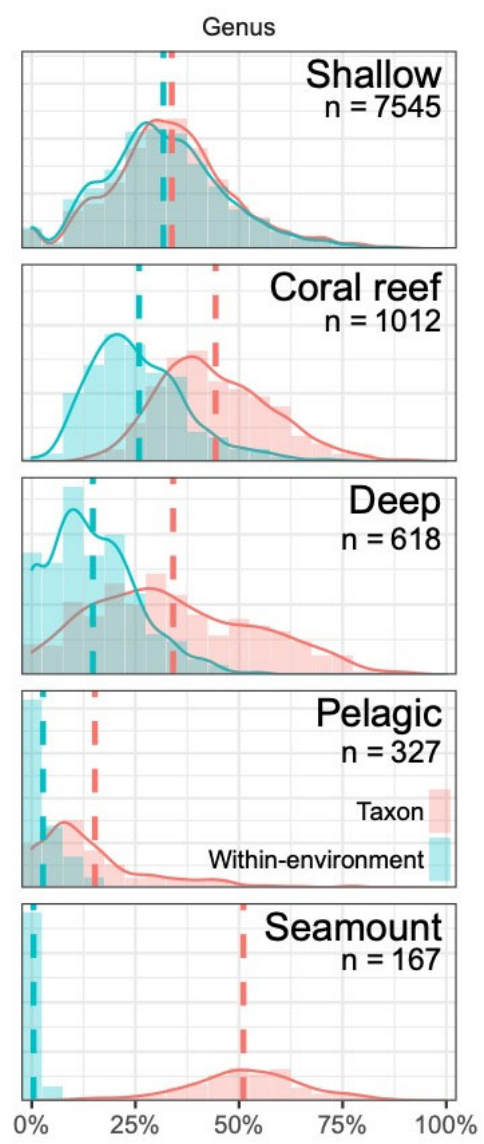

254 Supplementary Figure S3: Taxon (red) and within-environment (blue) fossilization potential 255 distributions of assemblages with environmental information $(n=9,669)$. Fossilization potential 256 calculated at order, family, and genus ranks. Histogram bin widths $=5 \%$, dashed lines indicate 257 mean. 

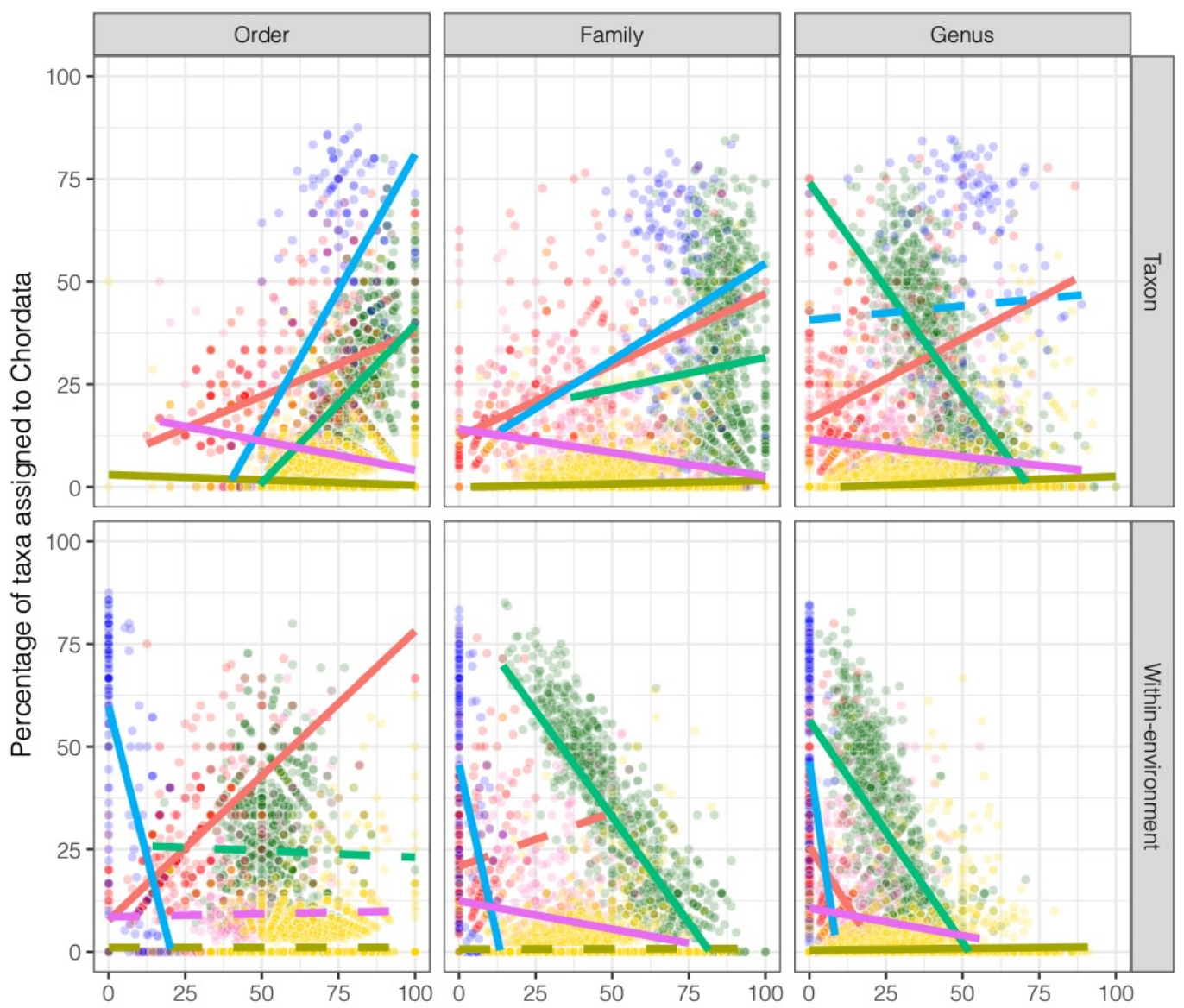

- Pelagic

- Shallow
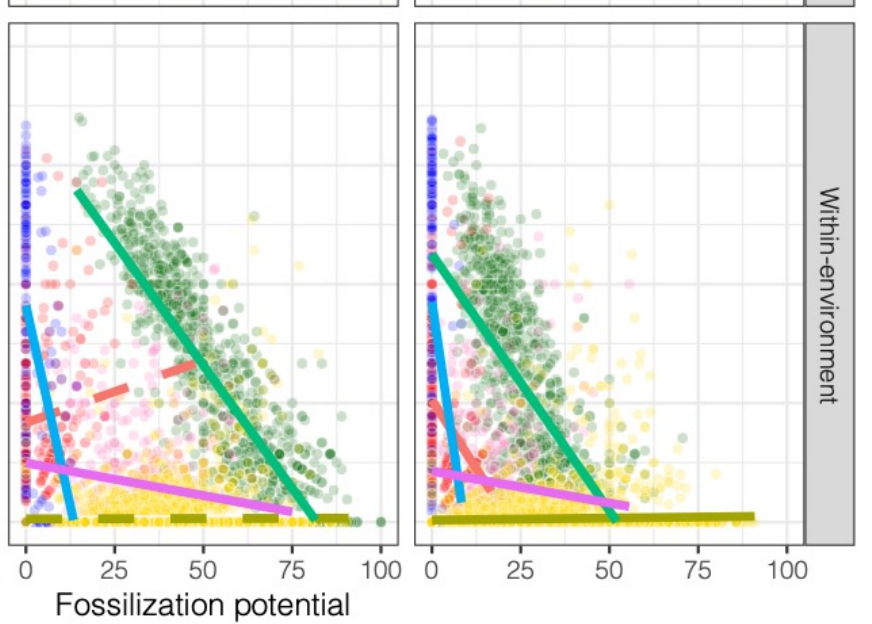

- Coral reef

- Seamount

- Deep

260 Supplementary Figure S4: Percentage of taxa (orders, families, or genera) in assemblages

261 belonging to the phylum Chordata, plotted against taxon and within-environment fossilization

262 potential. Datapoints indicate assemblages $(n=9,669)$ and are colored by environment. Solid

263 lines indicate significant linear regressions ( $p$-value $<0.05)$, dashed lines indicate insignificant 264 regressions. 


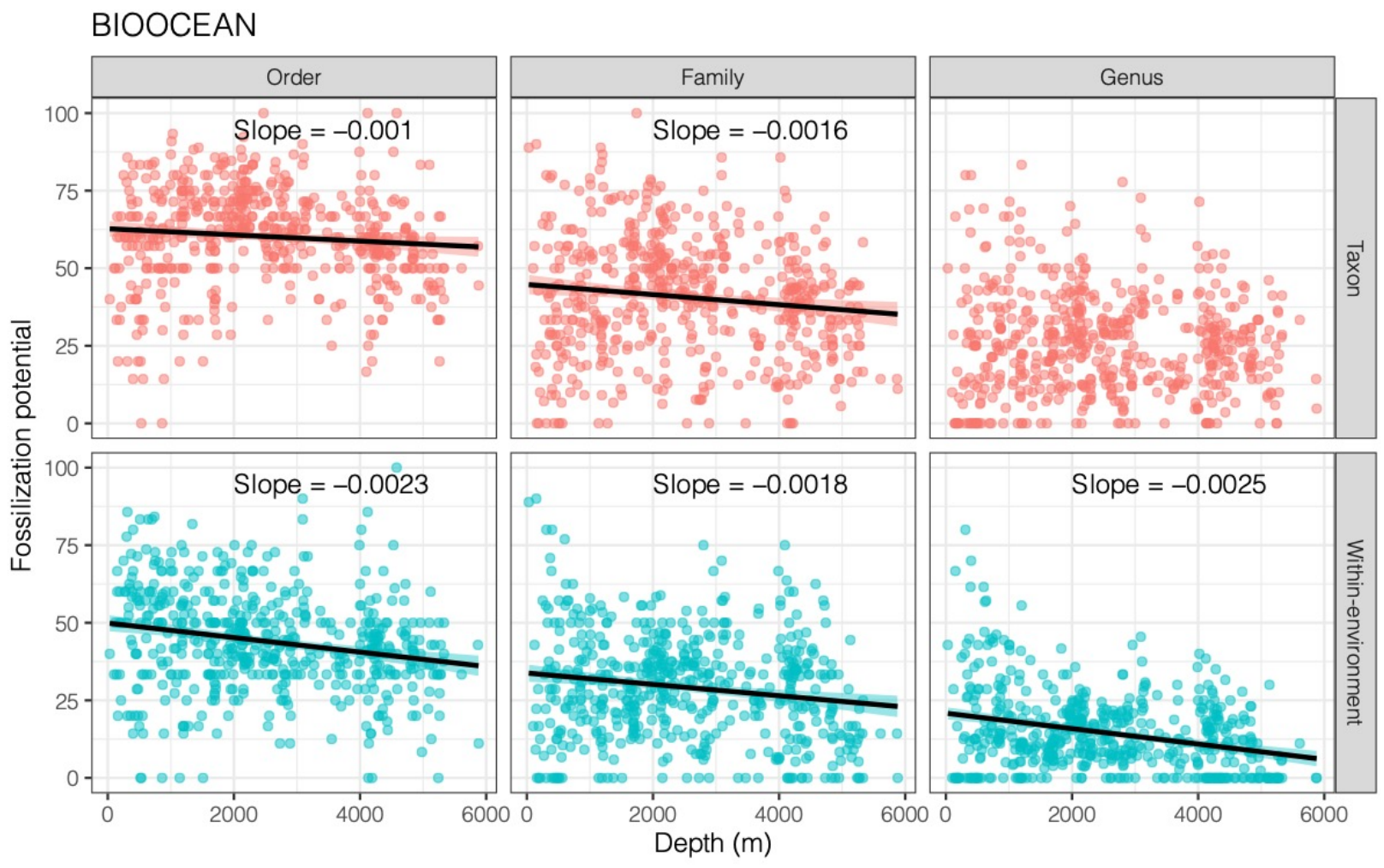

267 Supplementary Figure S5: Depth versus taxon (top) and within-environment (bottom)

268 fossilization potential for data from the BIOCEAN dataset $(n=549$; detailed in main text). Line

269 shows statistically significant linear regression with slope values indicated. 


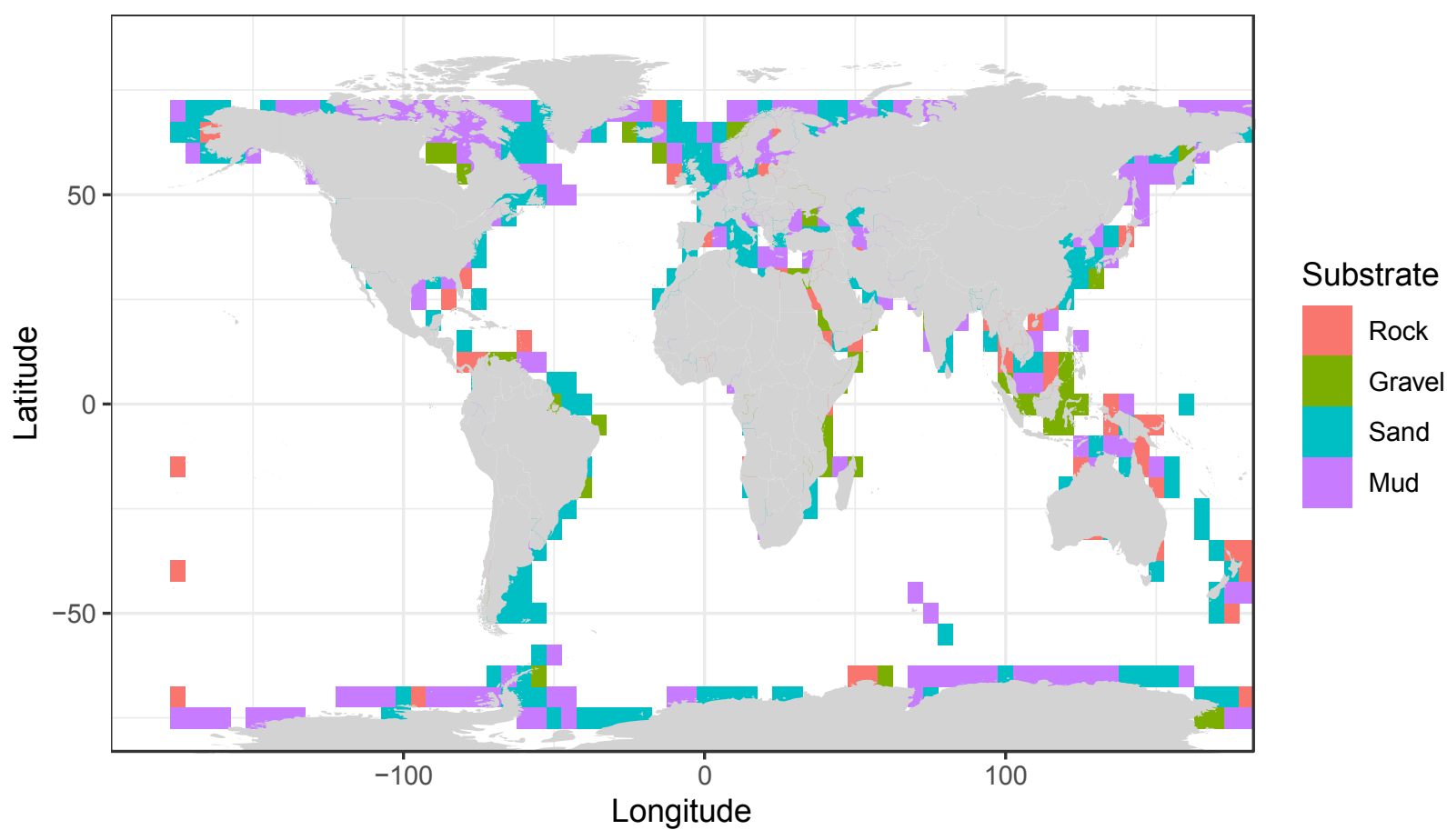

272 Supplementary Figure S6: Map of shallow water $(<1000 \mathrm{~m})$ substrate type interpolated using 273 dbSeabed data and KNN algorithm.

274 


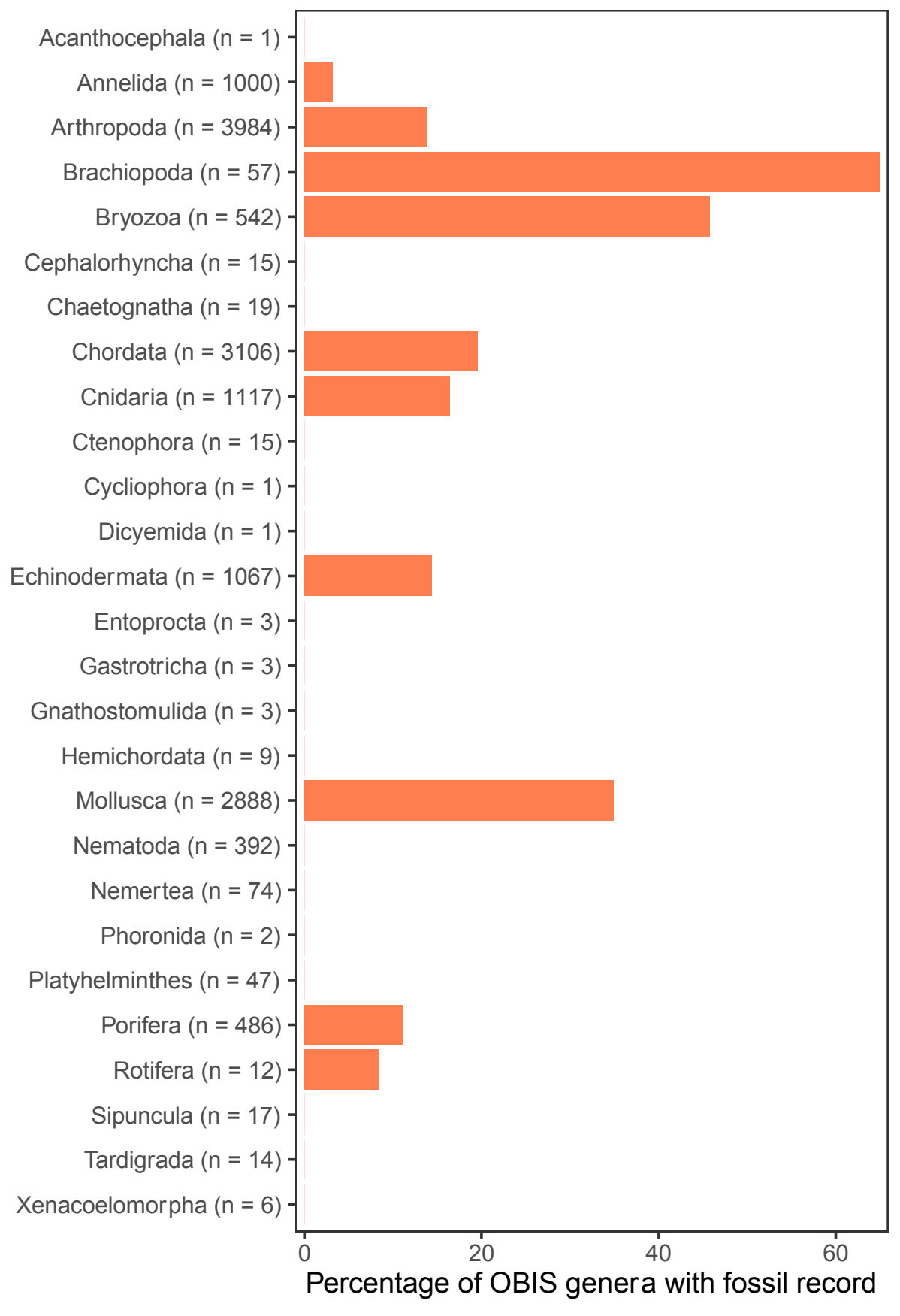

276 Supplementary Figure S7: Bar chart displaying the percentages of OBIS genera with records in 277 the Paleobiology Database (PBDB). The total numbers of OBIS genera are indicated in 278 parentheses. 

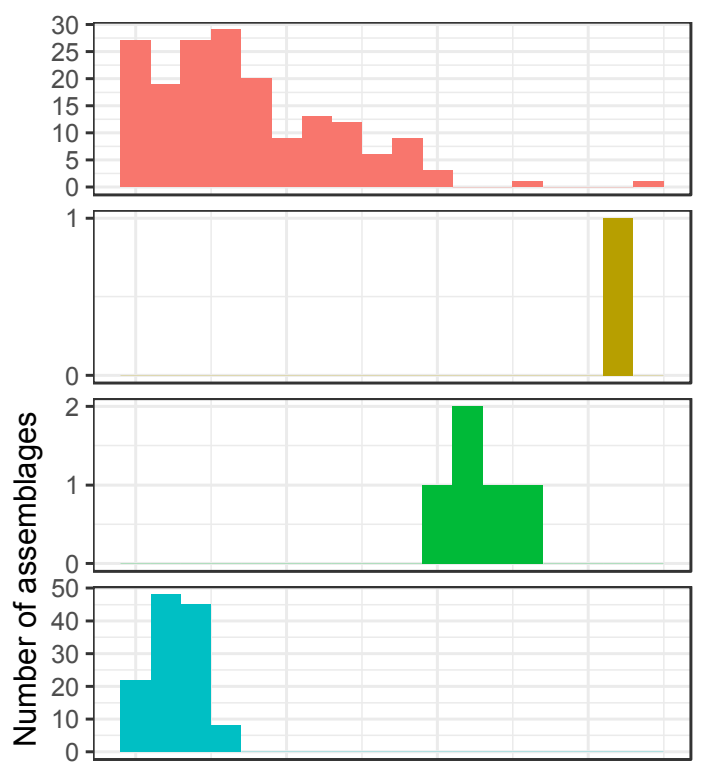

\section{Dataset title}
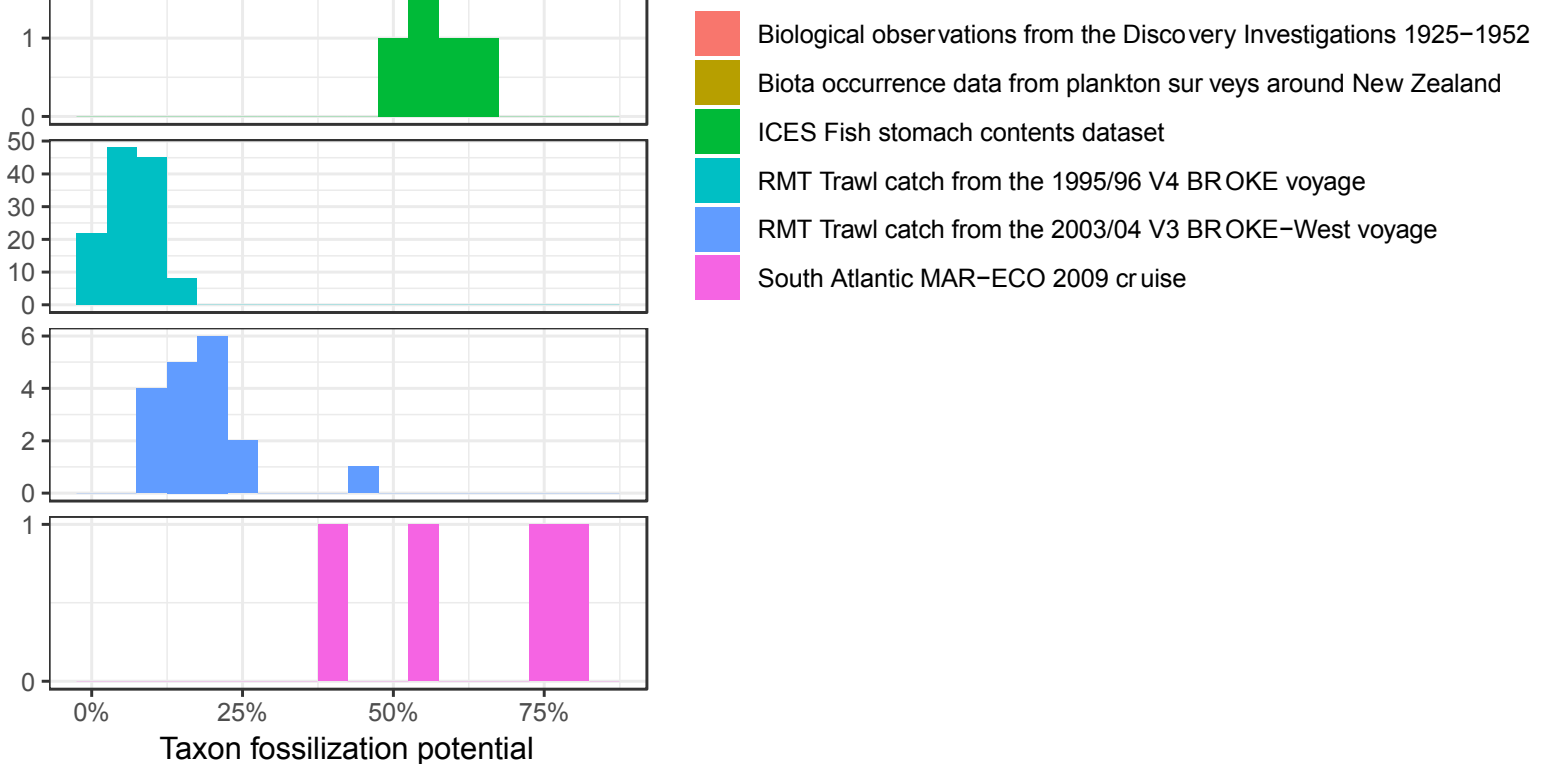

282 Supplementary Figure S8: Comparison of taxon fossilization potential distributions by dataset

283 ID $(n=6)$ for all pelagic communities $(n=327)$. 


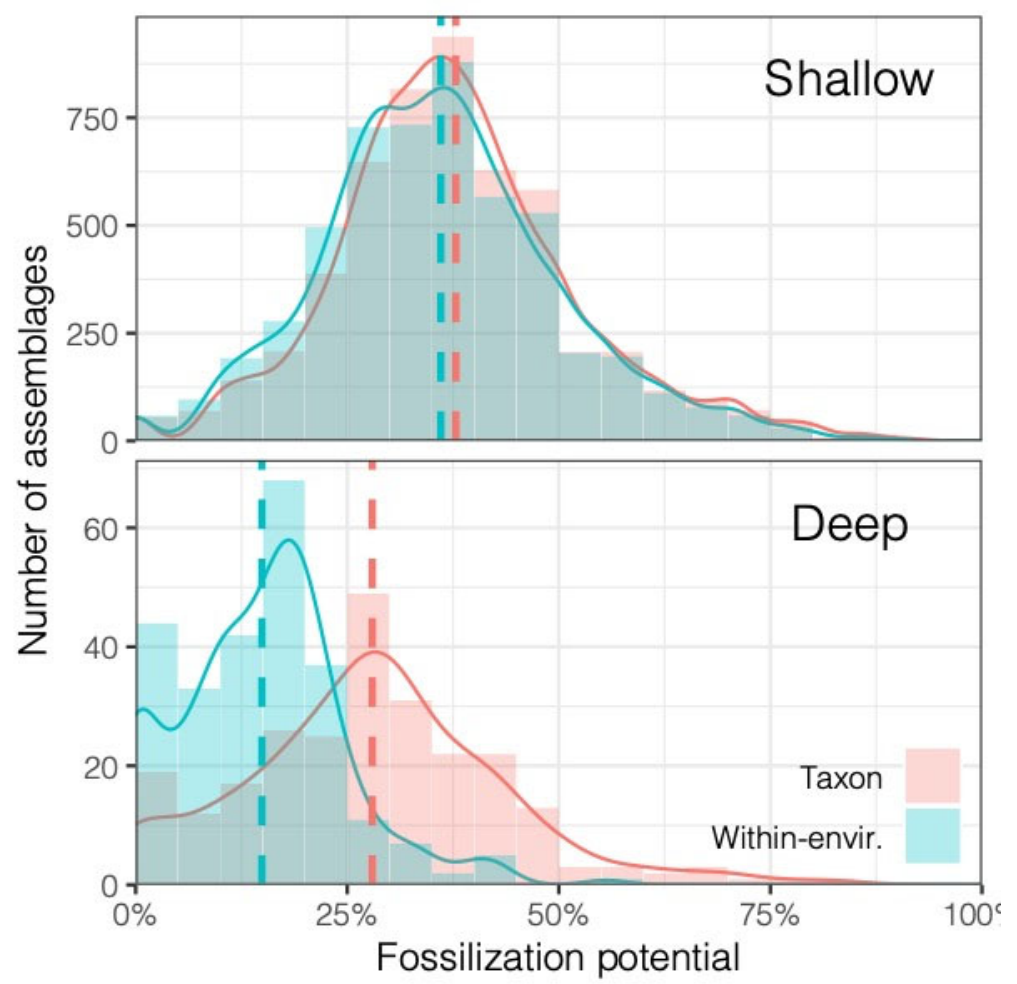

286 Supplementary Figure S9: Taxon and within-environment fossilization potential distributions 287 for assemblages within a bounded area in Europe $\left(58^{\circ} \mathrm{N}, 15^{\circ} \mathrm{W} ; 58^{\circ} \mathrm{N}, 22^{\circ} \mathrm{E} ; 35^{\circ} \mathrm{N}, 15^{\circ} \mathrm{W} ; 35^{\circ} \mathrm{N}\right.$, $28822^{\circ} \mathrm{E}$ ). Histogram bin widths $=5 \%$; dashed lines indicate mean. 

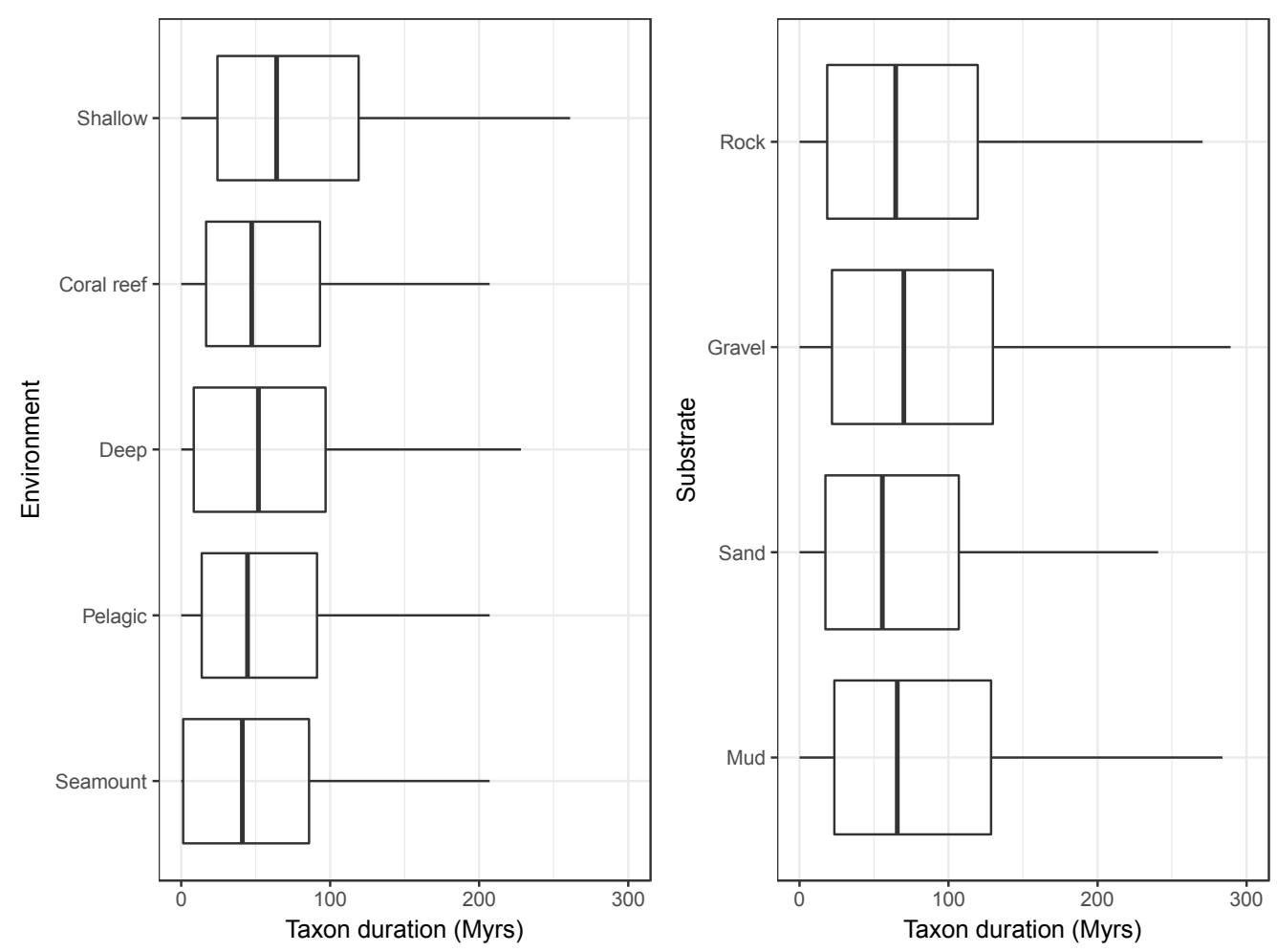

\begin{tabular}{|l|l|l|l|l|l|}
\hline & Lower whisker & Lower hinge & Median & Upper hinge & Upper whisker \\
\hline & & & & & \\
\hline Shallow & 0.01 & 24.36 & 64.06 & 119.12 & 261.10 \\
\hline Coral reef & 0.01 & 16.60 & 47.31 & 93.64 & 207.05 \\
\hline Deep & 0.01 & 8.47 & 51.90 & 96.89 & 228.06 \\
\hline Pelagic & 0.01 & 13.79 & 44.55 & 91.23 & 207.05 \\
\hline Seamount & 0.01 & 1.36 & 41.04 & 85.90 & 207.05 \\
\hline & & & & & \\
\hline Rock & 0.01 & 18.45 & 64.56 & 120.03 & 270.48 \\
\hline Gravel & 0.01 & 21.74 & 70.17 & 129.77 & 289.32 \\
\hline Sand & 0.01 & 17.32 & 55.63 & 107.12 & 240.76 \\
\hline Mud & 0.01 & 23.54 & 65.65 & 128.57 & 283.93 \\
\hline
\end{tabular}

293 Supplementary Figure S10A: Boxplots (top) and boxplot statistics (bottom) of genus durations

294 (calculation method outlined in supplementary information section "Taxon durations and

295 fossilization potential") for OBIS taxa with corresponding environment and substrate

296 information. Taxa without fossil evidence are excluded. Outliers removed for clarity. 

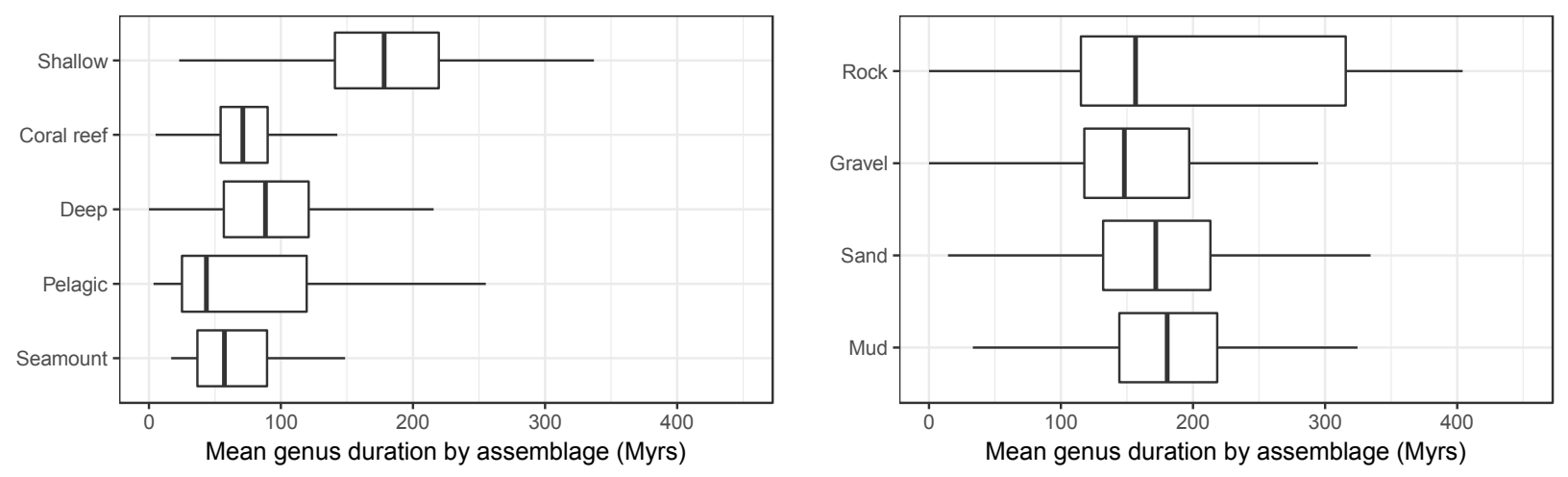

\begin{tabular}{|l|l|l|l|l|l|}
\hline & $\begin{array}{l}\text { Lower } \\
\text { whisker }\end{array}$ & Lower hinge & Median & Upper hinge & $\begin{array}{l}\text { Upper } \\
\text { whisker }\end{array}$ \\
\hline Shallow & 22.95 & 140.81 & 178.16 & 219.43 & 337.13 \\
\hline Coral reef & 5.08 & 54.30 & 71.19 & 89.94 & 142.76 \\
\hline Deep & 0.01 & 56.60 & 88.24 & 121.07 & 215.55 \\
\hline Pelagic & 3.49 & 25.02 & 43.52 & 120.13 & 261.92 \\
\hline Seamount & 16.78 & 36.74 & 57.06 & 89.51 & 148.55 \\
\hline Rock & 0.01 & 115.03 & 156.48 & 315.54 & 404.18 \\
\hline Gravel & 0.01 & 117.52 & 147.91 & 197.38 & 294.72 \\
\hline Sand & 14.39 & 132.00 & 171.75 & 213.15 & 334.38 \\
\hline Mud & 33.15 & 144.18 & 180.40 & 218.34 & 324.65 \\
\hline
\end{tabular}

301 Supplementary Figure S10B Boxplots (top) and boxplot statistics (bottom) of mean genus

302 duration values for assemblages with environment (top left) and substrate (top right) information.

303 Calculations of assemblage mean values only include the durations of taxa with fossil

304 representatives. Outliers removed for clarity. 


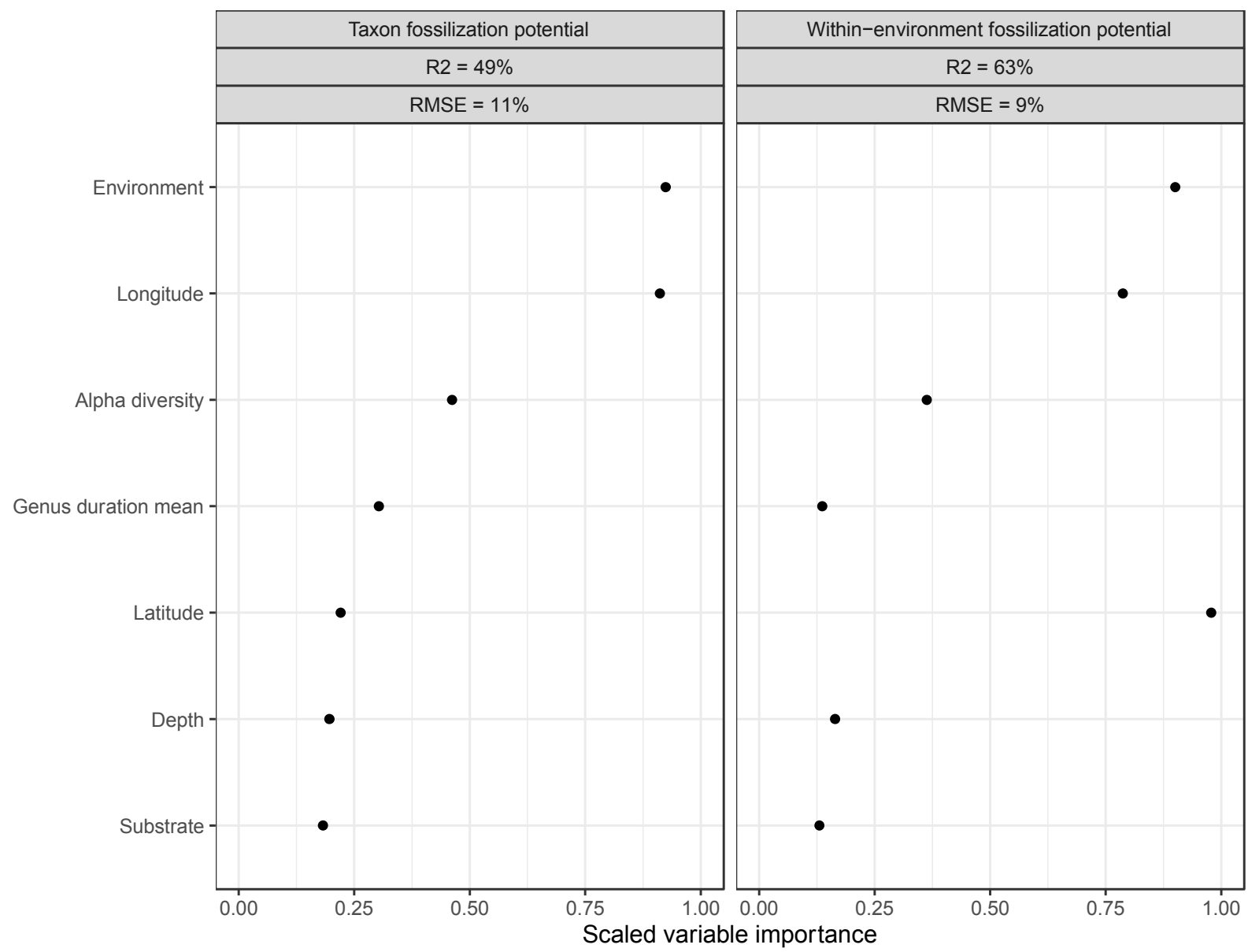

307 Supplementary Figure S11: Scaled variable importance scores for conditional inference

308 regression tree models predicting genus-level taxon and within-environment fossilization

309 potential. Importance rankings are based on the reduction in square error produced when the

310 variable is added. R-squared (R2) and root mean square error (RMSE) shown for each model. 


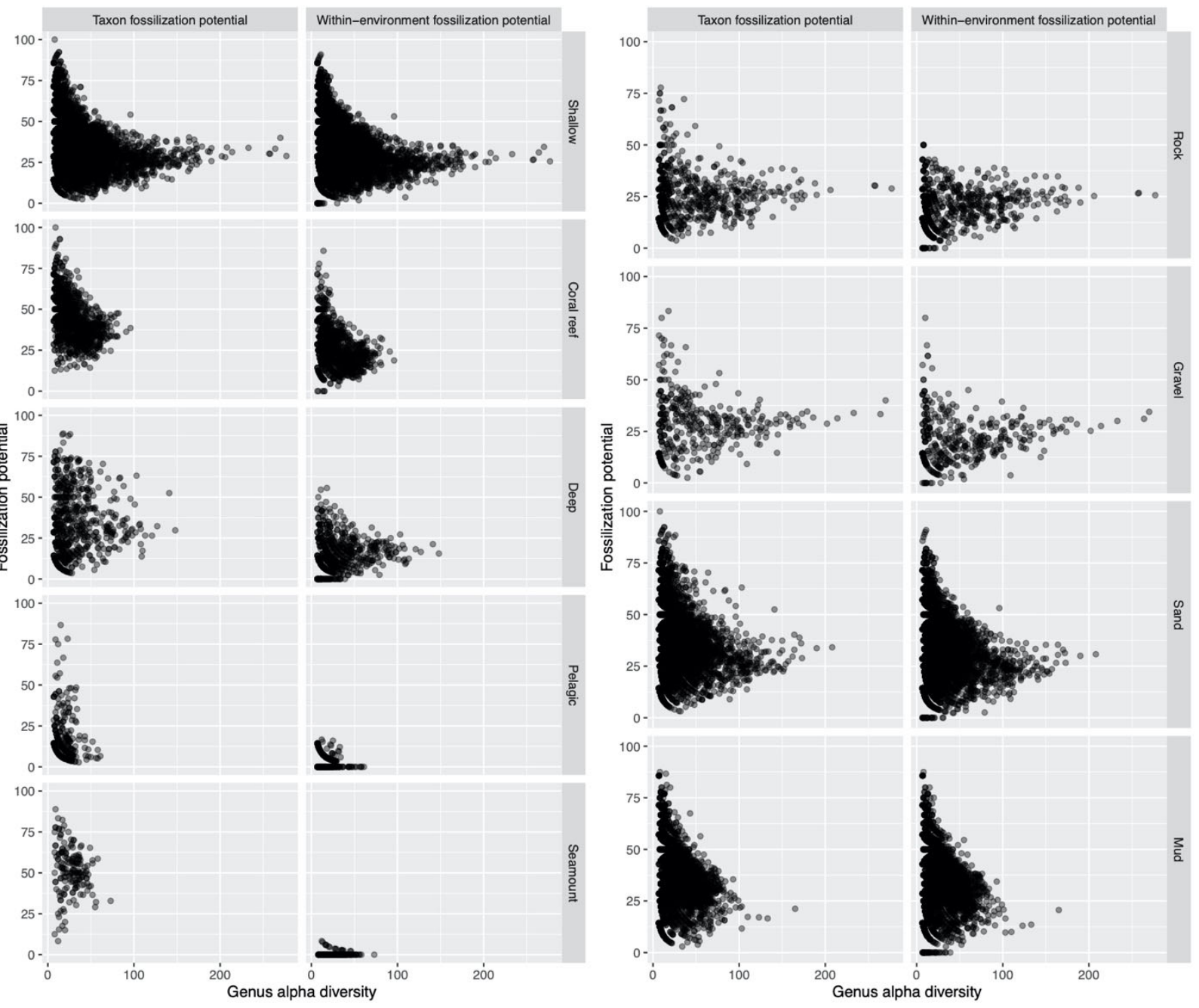

313 Supplementary Figure S12: Assemblage-specific genus richness for assemblages with

314 environment $(n=9,669)$ and substrate $(n=7,545)$ data plotted against fossilization potential. 


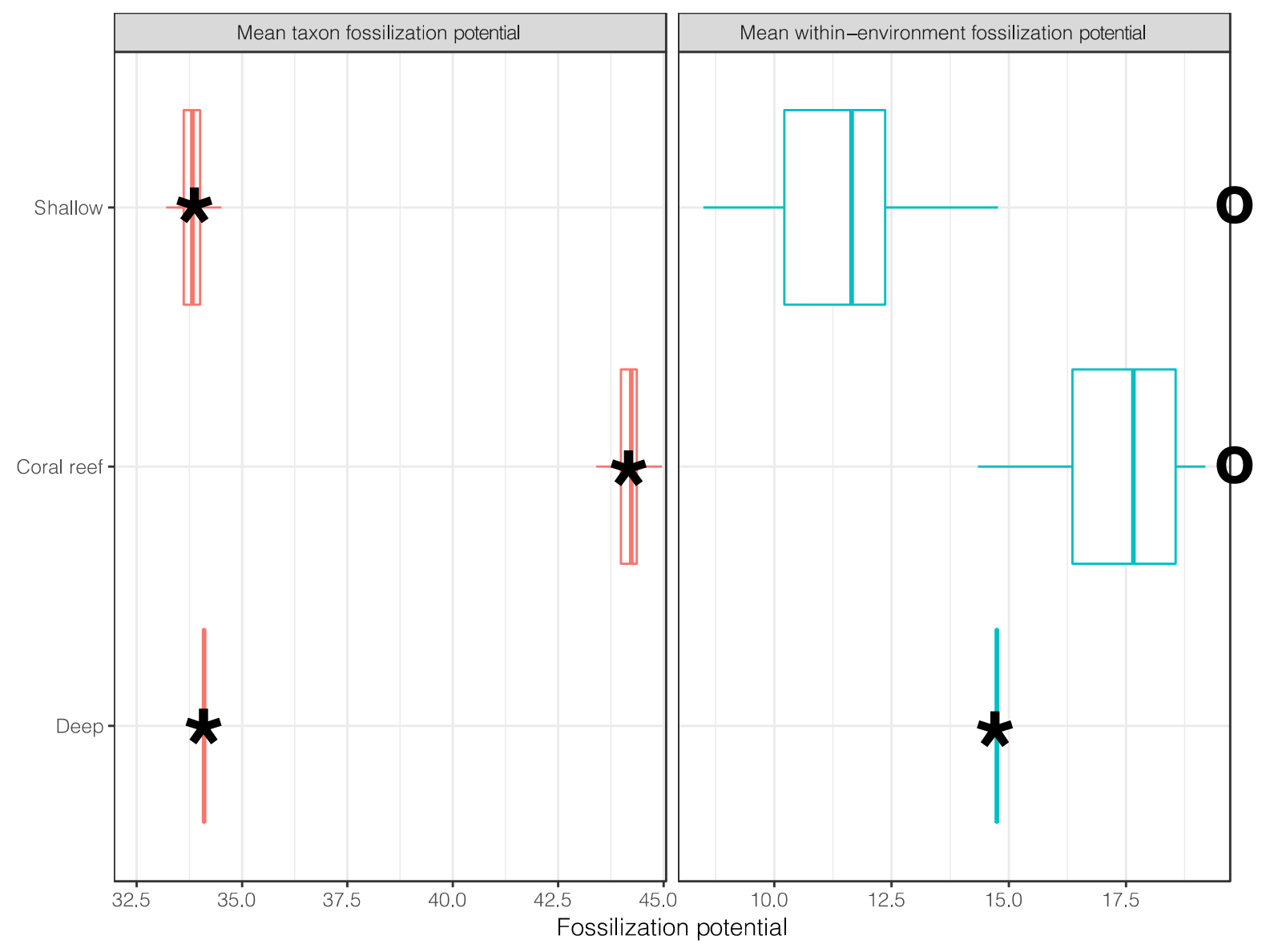

316 Supplementary Figure S13: Taxon and within-environment fossilization potential mean values

317 for subsampled data (repeated 1000 times). Each mean represents comparisons utilizing random

318 samples of PBDB taxa (5927 per environment, as per the smallest group, deep water) and

319 random samples of OBIS assemblages (1012 per environment, as per the smallest group, coral

320 reefs). Means of the raw data (e.g., Fig. 3) shown using black asterisks. Within-environment

321 fossilization potential for shallow and coral reef environments are off the axes (indicated by

322 circles) and have means of $32 \%$ and $26 \%$, respectively. 


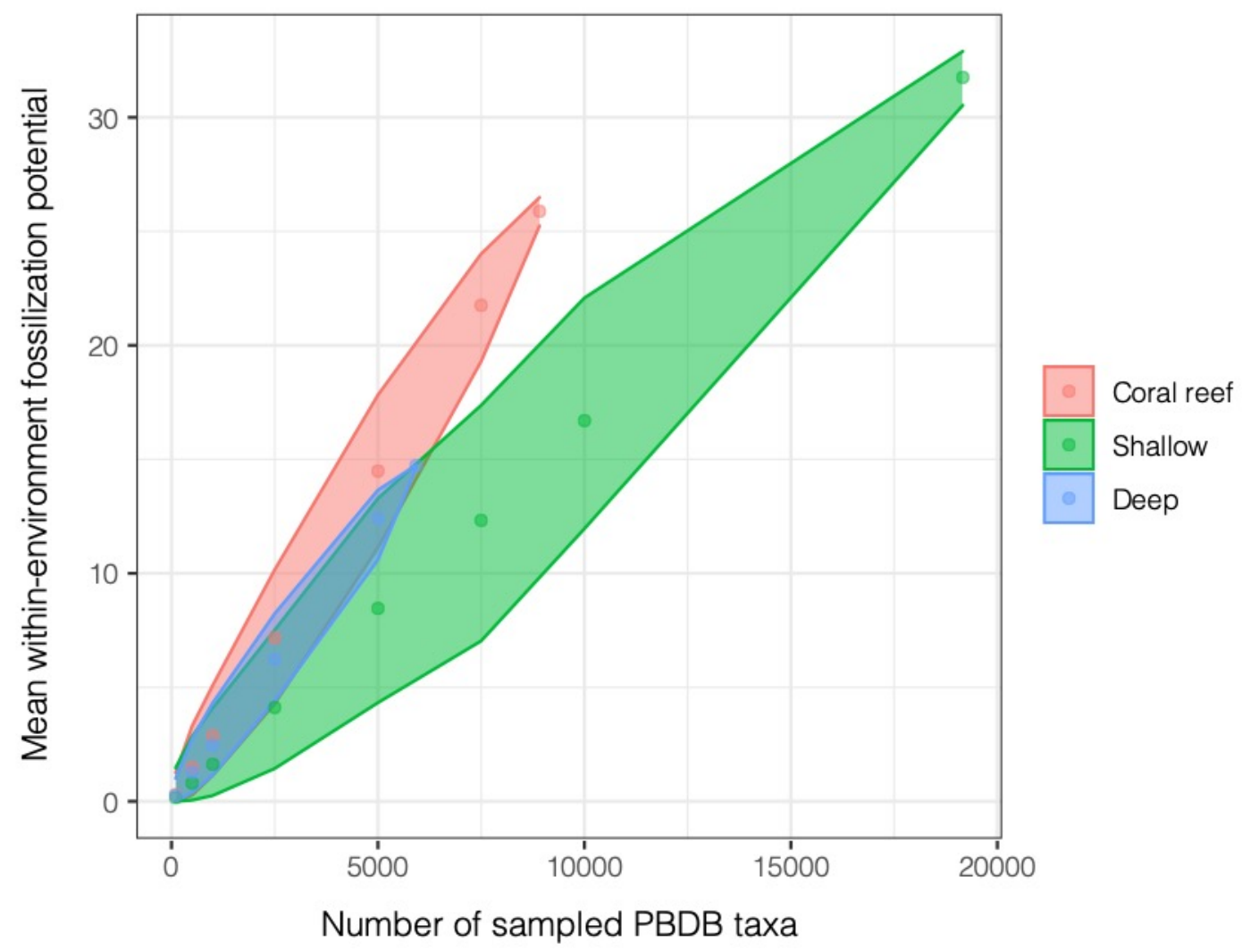

325 Supplementary Figure S14: 95\% confidence intervals of mean within-environment

326 fossilization potential values when calculated based on subsampled PBDB data. Mean within327 environment fossilization potential values were generated by first subsampling the number of 328 PBDB taxa per environment (x-axis), then comparing the number of OBIS taxa in an assemblage

329 to the subsampled PBDB faunal list to generate the assemblage-specific fossilization potential 330 value, and finally calculating the mean of all assemblage-specific fossilization potentials by 331 environment. This process was repeated 1000 times at a number of pre-defined PBDB sample 332 sizes $(100,500,1000,2500,5000,7500,10000,20000)$ up to the maximum number of PBDB 333 taxa assigned to each environment. 

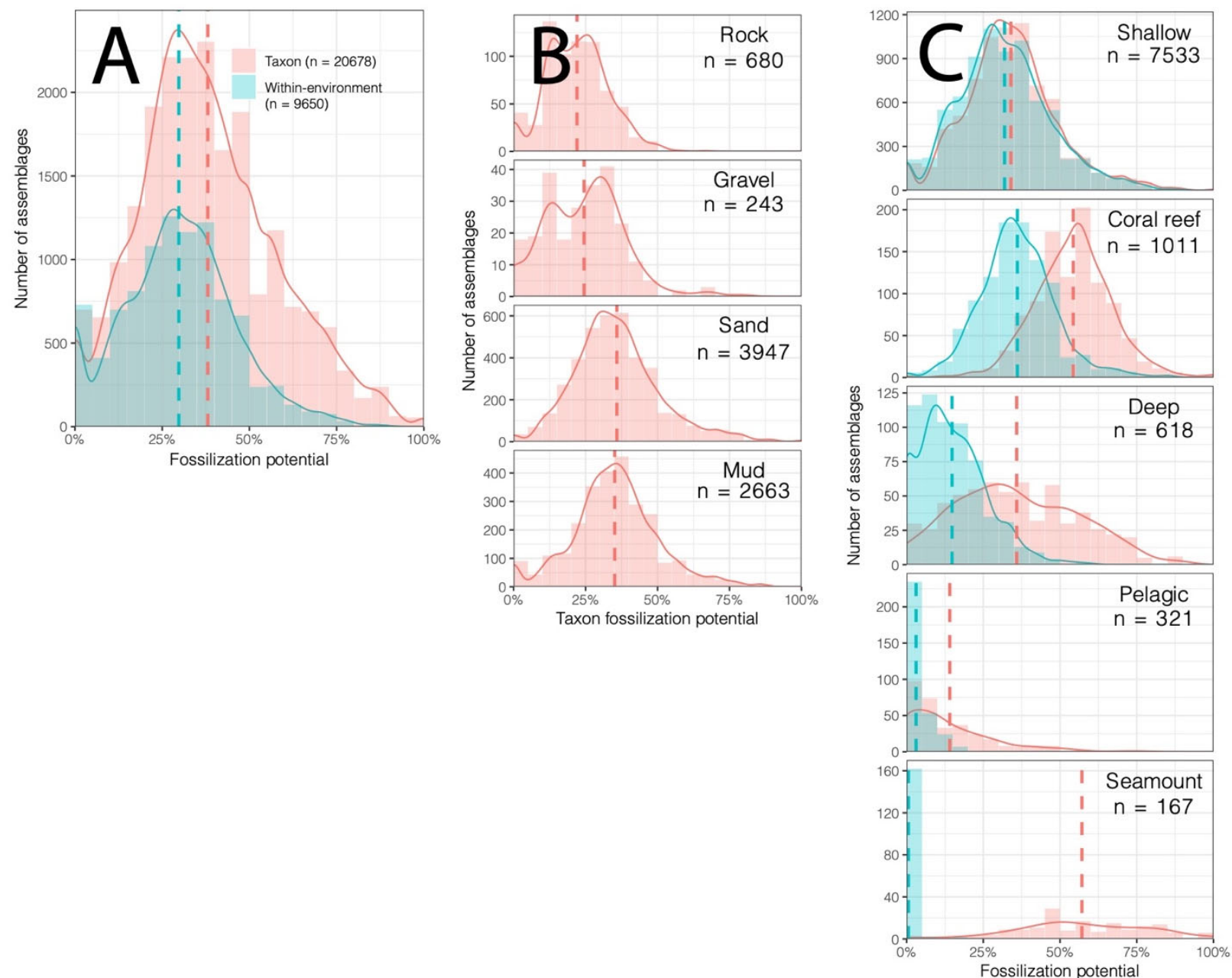

336 Supplementary Figure S15: Invertebrate only analyses. (A) Taxon fossilization potential and

337 within-environment fossilization potential (mean taxon/within-environment fossilization

338 potential $=39 / 30 \%$ ). (B) Taxon fossilization potential distributions for shallow water

339 assemblages with substrate information (mean fossilization potential values: rock $=22 \%$, gravel

$340=25 \%$, sand $=36 \%$, mud $=35 \%$ ). (C) Taxon and within-environment fossilization potential

341 distributions for assemblages with environmental information (mean taxon/within-environment

342 fossilization potential values: shallow $=33 / 30 \%$, coral reef $=53 / 35 \%$, deep $=35 / 14 \%$, pelagic $=$

$34314 / 2 \%$, seamount $=57 / 0 \%$ ). Histogram bin widths $=5 \%$; dashed lines indicate mean. 


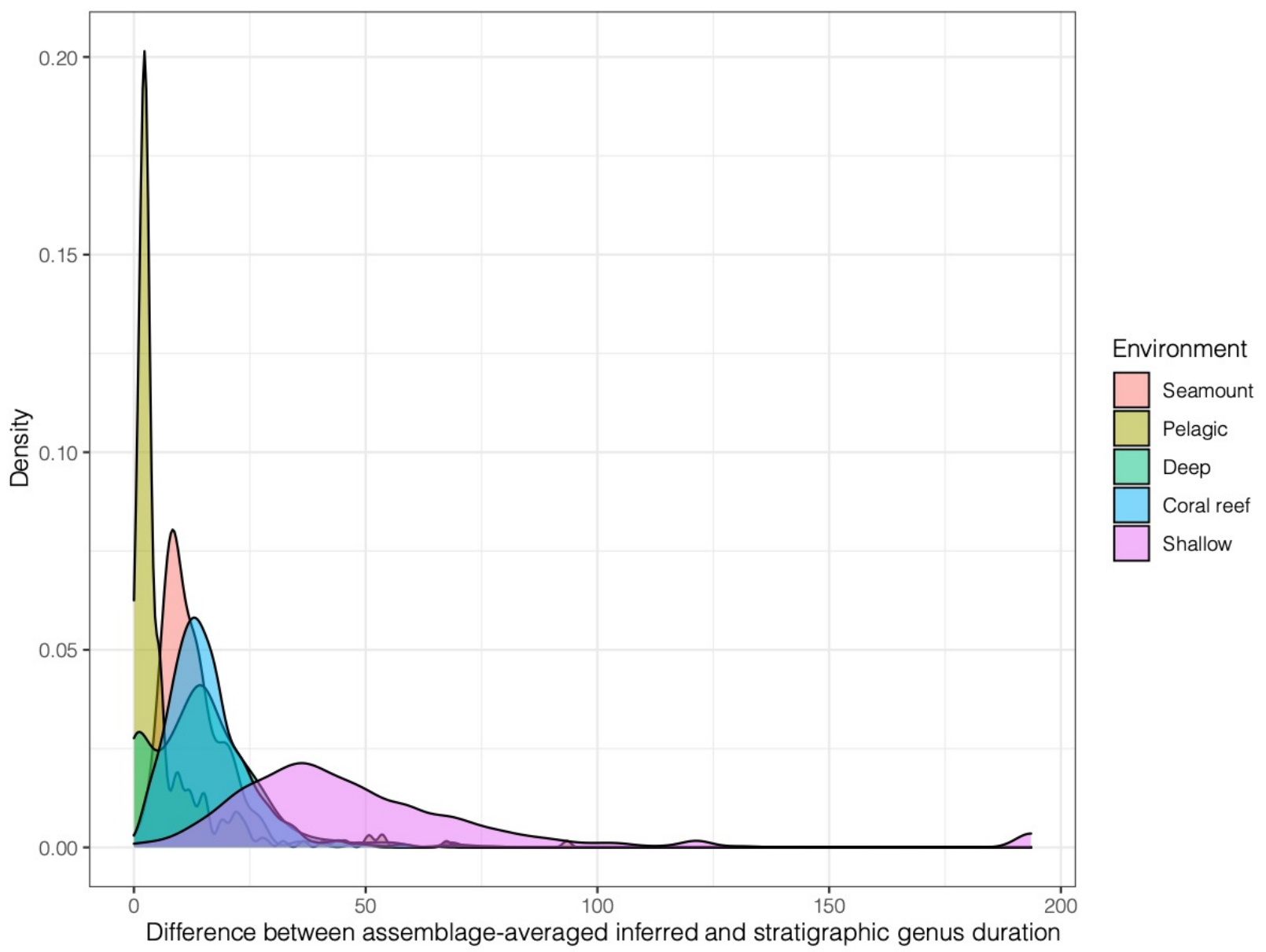

346 Supplementary Figure S16: Density plot of differences between assemblage averaged inferred347 and PBDB-durations parsed by environment. 
349 Supplementary data are available for download at the Harvard Dataverse Repository

350 (https://doi.org/10.7910/DVN/MMMTYZ). Supplementary Data labels and descriptions apply to 351 data files listed in the Dataverse Repository.

352 Supplementary Data DR1: Raw list of all occurrences in OBIS downloaded on $21^{\text {st }}$ January 353 2020. Occurrences were culled (procedures in text) using R code (Sup. Data DR4A). Column 354 headers following Darwin Core format (Wieczorek et al., 2012).

355 Supplementary Data DR2: Raw list of OBIS datasets (downloaded $20^{\text {th }}$ January 2020, Sup. 356 Data 2A) and list of OBIS assemblages (Sup. Data DR2B) meeting the culling procedures 357 described herein and applied using R Code (Sup. Data DR4A).

To generate assemblages, we removed occurrences from Sup. Data 1 (i) lacking data on genus, latitude, longitude, depth, or duration of sampling, (ii) from non-marine settings, or (iii) with inadequate error control (e.g., citizen science data). Occurrences were grouped into assemblages if they were obtained within $0.1^{\circ}$ latitude and $0.1^{\circ}$ longitude of each other, in the same $10 \mathrm{~m}$ depth bin, during the same year, and from the same dataset. We retained only assemblages with representatives of at least three phyla, four classes, five orders, six families, and seven genera in order to avoid poorly sampled assemblages. Alternative binning procedures were tested and did not affect interpretations (Figs. S1).

Additional assemblage data (id_habitat, id_scope, id_plankton, id_sampling, id_fishery, and id_environment) in Sup. Data DR2B was manually assigned to datasets using information from dataset titles and abstracts in Sup. Data DR2A. Column headers in Sup. Data DR2B are as follows: "dataset.par" = unique depth-, year-, location-, and dataset-specific assemblage identifier; "dataset_id" = an identifier for the set of data, which may be a global unique identifier or an identifier specific to a collection or institution; "depth.slice" $=10 \mathrm{~m}$ depth bin of assemblage; "LongBin" $=0.1^{\circ}$ longitudinal bin of assemblage; "LatBin" $=0.1^{\circ}$ latitudinal bin of assemblage; "id_habitat" = marine realm of dataset, pelagic or benthic; "id_scope" = taxonomic

374 scope of dataset; "id_plankton" = plankton-only dataset; "id_sampling" = sampling type of

375 assemblage; "id_environment" = environment type of dataset; "title" = name of dataset; "abstract" = abstract of dataset. 
377 Supplementary Data DR3: List of all occurrences in PBDB with select columns, downloaded 378 on $17^{\text {th }}$ January 2020 (A). List of Lagerstätten names generated by manually searching for names 379 in PBDB ("formation") based on the list of Lagerstätten in Muscente et al. (2017) (B).

380 Data downloaded using the following URL:

381 http://paleobiodb.org/data1.2/occs/list.csv?datainfo\&rowcount\&base_name=Animalia\&show=fu 38211 ,genus,env

383 Supplementary Data DR4: R Code used for data cleaning (A), identifying assemblages and 384 calculating fossilization potential (B), analyzing fossilization potential (C), generating genus 385 duration estimates (D; Wang et al., 2016), applying genus duration estimate calculations to OBIS taxa $(\mathrm{E})$, and predicting fossilization potential using conditional inference tree models $(\mathrm{F})$.

387 Supplementary Data DR5: Seafloor substrate data (Jenkins, 2008; A), code used to create 388 predictive model (B), and predictive model (native R format, “.RDS”) applied to OBIS assemblages to assess substrate type (C).

Substrate data (Sup. Data DR5A) used for interpolation were supplied by Jenkins (personal communications): "wd_vals" = water depth; "lat" = latitude, to two decimal places; 392 "lon" = longitude, to two decimal places; "substrate" = dominant substrate type (rock, gravel, 393 sand, and mud).

We interpolated substrate data based on the dbSeabed dataset (Jenkins, 2008) using a k395 nearest neighbors (KNN) algorithm. The KNN algorithm is a non-parametric supervised machine 396 learning algorithm used for classification and regression. The KNN predictive model was built 397 using the R package KKNN (Schliep, Hechenbichler, and Lizee, 2016). We performed leave398 one-out cross-validation for computationally efficient training of the predictive model. We tested 399 K-values ranging 1-20 and all possible kernel types ("rectangular", "triangular", "epanechnikov", 400 "gaussian", "rank", and "optimal"). For training, dbSeabed data was split so that $80 \%$ of the data 401 was used to train the model and $20 \%$ to test it. Training identified a K-value of 12 and a 402 triangular kernel as the best parameters, although kernel type did not alter resultant 403 classifications. Our best model had a k-value of 12 and a triangular kernel shape, meaning that 
404 the 12-nearest neighbors within a triangle shaped space of the focal grid cell were used to infer 405 the substrate type assigned to that grid cell.

406 Prior to analysis, we extended the dbSeabed data by 10 degrees of longitude on either

407 side (i.e., so that the map spanned -190 to 190 degrees). This allowed the predictive model to 408 utilize data at the edge of its conventional range (-180 to 180 degrees). If this correction was not 409 applied, the model would not know that data at -179 degrees longitude and 179 degrees longitude 410 are proximal. To extend the map, we simply duplicated data in the range of -180 to -170 degrees 411 and 170 to 180 degrees by adding and subtracting, respectively, 360 degrees (i.e., -179 became 412181 degrees longitude).

413 Supplementary Data DR6: PBDB environments matched to broader categories for comparison 414 with OBIS designations.

415 Supplementary Data DR7: Tests of sampling biases for shallow water, coral reef, and deep 416 water assemblages (see supplementary information section "Sampling biases" for more 417 information). 


\section{REFERENCES}

Briggs, D.E.G., Erwin, D.H., and Collier, F.J., 1994, The fossils of Burgess Shale: Washington DC, Smithsonian Institution Press.

Crampton, J.S., Cooper, R.A., Beu, A.G., Foote, M., and Marshall, B.A., 2010, Biotic influences on species duration: interactions between traits in marine molluscs: Paleobiology, v. 36, p. 204-223, doi:https://doi.org/10.1666/09010.1.

Fortey, R.A., 1980, Generic longevity in lower ordovician trilobites: Relation to environment: Paleobiology, v. 6, p. 24-31, doi:https://doi.org/10.1017/S009483730001246X.

Horton, T. et al., 2020, World Register of Marine Species (WoRMS): https://www.marinespecies.org (accessed January 2020).

Hothorn, T., and Zeileis, A., 2015, partykit : A Toolkit for Recursive Partytioning: Journal of Machine Learning Research, v. 16, p. 3905-3909.

Hou, X., Siveter, D.J., Siveter, D.J., Aldridge, R.J., Cong, P., Gabbott, S.E., Ma, X., Purnell, M.A., and Williams, M., 2017, The Cambrian Fossils of Chengjiang, China: John Wiley \& Sons, doi:https://doi.org/10.1002/9781118896372.

Jablonski, D., 2008, Species Selection: Theory and Data: Annual Review of Ecology, Evolution, and Systematics, v. 39, p. 501-524, doi:https://doi.org/10.1146/annurev.ecolsys.39.110707.173510.

Jablonski, D., and Bottjer, D.J., 1983, Soft-bottom epifaunal suspension-feeding assemblages in the Late Cretaceous: implications for the evolution of benthic paleocommunities., in Biotic interactions in Recent and fossil benthic communities, p. 747-812.

Jablonski, D., and Hunt, G., 2006, Larval ecology, geographic range, and species survivorship in cretaceous mollusks: Organismic versus species-level explanations: American Naturalist, v. 168, p. 556-564, doi:https://doi.org/10.1086/507994.

Jenkins, C., 2008, dbSEABED: an information processing system for marine substrates:, https://instaar.colorado.edu/ jenkinsc/dbseabed/ (accessed April 2020). 
444 Kammer, T.W., Baumiller, T.K., and Ausich, W.I., 1997, Species longevity as a function of 445 niche breadth: Evidence from fossil crinoids: Geology, v. 25, p. 219-222, 446 doi:https://doi.org/10.1130/0091-7613(1997)025<0219:SLAAFO>2.3.CO;2.

447 Muscente, A.D. et al., 2017, Exceptionally preserved fossil assemblages through geologic time 448 and space: Gondwana Research, v. 48, p. 164-188, 449 doi:https://doi.org/10.1016/J.GR.2017.04.020.

450 Schliep, K., Hechenbichler, K., and Lizee, A., 2016, kknn: Weighted k-Nearest Neighbors:, 451 https://cran.r-project.org/web/packages/kknn/index.html (accessed April 2020).

452 Wang, S.C., Everson, P.J., Zhou, H.J., Park, D., and Chudzicki, D.J., 2016, Adaptive credible 453 intervals on stratigraphic ranges when recovery potential is unknown: Paleobiology, v. 42, 454 p. 240-256, doi:https://doi.org/10.1017/pab.2015.37.

455 Wieczorek, J., Bloom, D., Guralnick, R., Blum, S., Döring, M., Giovanni, R., Robertson, T., and 456 Vieglais, D., 2012, Darwin core: An evolving community-developed biodiversity data 457 standard: PLoS ONE, doi:https://doi.org/10.1371/journal.pone.0029715. 